\title{
Partitioning of ribonucleoprotein complexes from the cellular actin cortex
}

C. Isaac Angert ${ }^{1}$, Siddarth Reddy Karuka ${ }^{1}$, Louis M. Mansky ${ }^{2,3,4}$, Joachim D. Mueller ${ }^{1,2,5}$

School of Physics and Astronomy ${ }^{1}$, University of Minnesota, Minneapolis, MN 55455

Institute of Molecular Virology2, University of Minnesota, Minneapolis, MN 55455

Division of Basic Sciences, School of Dentistry ${ }^{3}$, University of Minnesota, Minneapolis, MN 55455

Masonic Cancer Center ${ }^{4}$, University of Minnesota, Minneapolis, MN 55455

Department of Biomedical Engineering ${ }^{5}$, University of Minnesota, Minneapolis, MN 55455

\section{Abstract}

13 The cell cortex plays a crucial role in cell mechanics, signaling, and development. However, little is known

14 about the influence of the cortical meshwork on the spatial distribution of cytoplasmic biomolecules. Here, 15 we describe a new fluorescence microscopy method to infer the intracellular distribution of labeled 16 biomolecules with sub-resolution accuracy. Unexpectedly, we find that RNA-binding proteins are partially 17 excluded from the cytoplasmic volume adjacent to the plasma membrane that corresponds to the actin cortex. Complementary diffusion measurements of RNA-protein complexes suggest that a rudimentary model based on excluded volume interactions can explain this partitioning effect. Our results suggest the actin cortex meshwork may play a role in regulating the biomolecular content of the volume immediately adjacent to the plasma membrane.

\section{Teaser}

A novel microscopy technique reveals partial exclusion of RNA-protein complexes from the actin cortex due to their large hydrodynamic size.

\section{Introduction}

Both the actin cortex and RNA are fundamental building blocks of eukaryotic cells. The cortex, acting in concert with other cytoskeletal elements, is involved in nearly all aspects of cell mechanics, such as cell structure, membrane rigidity, migration and division (1). Due to its location adjacent to the plasma membrane, the cortex plays important roles in signal transduction and mechanical response pathways $(1$, 2). In contrast, RNA molecules serve as genomic messengers and constitute major components of the cellular translational machinery. Specific localization of mRNA to sub-cellular regions has been widely observed (3). RNA localization to sub-cellular compartments such as the endoplasmic reticulum (ER), 
processing $(\mathrm{P})$-bodies or distal cytoplasm in neurons is critical to aspects of development (4) and is an important regulator of cellular function (5). Due to their highly structured and extended nature, mRNAs have hydrodynamic radii that are dramatically larger than those of the globular proteins they encode (6). Based on a recent study $(7)$, a typical mRNA of $1-2$ kilobase length $(8,9)$ is expected to have a hydrodynamic radius in the range of $\sim 10 \mathrm{~nm}$. The radii of ribosomes are of comparable size $(10-15 \mathrm{~nm})(10,11)$.

Previous biophysical studies have established that the cell cytoplasm is a complex medium $(12,13)$ that modulates the diffusion and localization of large $(>10 \mathrm{~nm})$ particles in a size-dependent manner (14-18). These observations have prompted the question of whether the large size of the cellular translational machinery might broadly influence its subcellular localization (19). Here, the novel observation that large riboneucleoprotein (RNP) complexes are partially excluded, or partitioned, from the interior of the actin cortex is reported. These observations are facilitated by recent developments in two-photon dual-color (DC) z-scan fluorescence microscopy (20), which is adapted in the present work to infer protein localizations inside living cells with sub-diffraction resolution. DC z-scans are acquired by moving the two-photon excitation volume vertically through the cytoplasm of adherent cells. This movement of the excitation volume yields fluorescent intensity traces that encode the vertical intracellular distribution of a fluorescently labeled protein species as well as that of a distinctly colored reference fluorescent protein. Although different reference species are possible, our typical choice is a soluble, purely cytoplasmic protein, which serves as a "paint" that marks the cytoplasmic extent. Differential vertical localization between the fluorescently labeled protein and the reference protein is encoded as subtle differences in the shape of the DC z-scan traces. The magnitude of the differential vertical localization is quantitatively recovered from the data by fitting the z-scan traces (20).

\section{Results}

Initial proof-of-principle experiments were conducted with U2OS cells to demonstrate the utility of DC z-scan to quantify differences in intracellular protein distributions. For detailed descriptions of the z-scan model geometries used in the following, see the Materials and Methods section. First, cells were co-transfected with mCherry-RXR (a protein that localizes in the nucleus) and EGFP (which diffuses freely throughout the cell). DC z-scans through the cell nucleus were fit to the $S^{G}-S^{C H}$ (Eq. 17) model to identify the slab length associated with the mCherry fluorescence, which corresponds to the nuclear thickness, and the slab length of the EGFP signal, which corresponds to the slightly longer thickness of the entire cell (Fig. 1A). A representative z-scan with fit shows that the mCherry-RXR and EGFP z-scans are well described by this 
model, with flat residuals and $X_{v}{ }^{2}=1.2$ (Fig. 1B). The fit identified the intensity amplitudes of mCherry-RXR and EGFP, the locations of the bottom and top cell edges $\left(a^{G}=2.16 \mu \mathrm{m}\right.$ and $\left.b^{G}=5.80 \mu \mathrm{m}\right)$, as well as the locations of the bottom and top nuclear edges $\left(a^{C H}=2.51 \mu \mathrm{m}\right.$ and $\left.b^{C H}=5.03 \mu \mathrm{m}\right)$, respectively. The distances between the edges of the EGFP and mCherry slab were defined at the top and bottom of the cell by $\Delta^{T}=b^{C H}-b^{G}=-770 \mathrm{~nm}$ and $\Delta^{B}=a^{G}-a^{C H}=-350 \mathrm{~nm}$, respectively. Since $\Delta$ measures the displacement between the borders of the EGFP and mCherry layers, its value can be positive or negative in general. Distances are reported by taking the absolute value $|\Delta|$, which in this instance determines the thickness of the cytoplasmic layers surrounding the top and bottom of the nucleus (Fig. 1A). Repeated DC z-scan experiments on multiple cells yielded multiple estimates of $\Delta^{T}$ and $\Delta^{B}$ which are summarized by a histogram (Fig. 1C) with averages of $\left\langle\Delta^{\top}\right\rangle=-340 \pm 50 \mathrm{~nm}$ and $\left\langle\Delta^{B}\right\rangle=-260 \pm 30 \mathrm{~nm}(\mathrm{SEM}, n=19)$.

As a control, DC z-scans were performed through the cytoplasm of U2OS cells co-expressing EGFP and mCherry fluorescent proteins (Fig. 1D). Since both proteins are soluble species within the cell cytoplasm, DC z-scans are expected to recover $\Delta=0$, on average. As shown in Fig. 1E, a representative DC z-scan fits to a $S^{G}-S^{C H}$ (Eq. 17) model with flat residuals and $X v^{2}=1.1$. Repeated $D C$-scans in multiple cells recovered $\left\langle\Delta^{B}>=-2.3 \pm 1.2 \mathrm{~nm}\right.$ and $\left\langle\Delta^{T}\right\rangle=1.0 \pm 3.1 \mathrm{~nm}$ (SEM, $\left.n=59\right)$. The histogram of the combined displacements is shown in Fig. 1F. For the EGFP and mCherry co-expression, the weighted average of the combined data yielded $\langle\Delta>=-0.5 \pm 1.7 \mathrm{~nm}$ (SEM, $n=118$ ), which is consistent with $\Delta=0 \mathrm{~nm}$ within $0.3 \mathrm{SD}$. Note that since the diameter of globular proteins is typically several nanometer, differences of less than $10 \mathrm{~nm}$ were considered insignificant in this study and treated as consistent with zero.

Next, DC z-scans were performed in U2OS cells co-expressing EGFP-HRas and mCherry (Fig. 1G). EGFP-HRas was previously reported to be a peripheral membrane protein that binds to the plasma membrane as a monomer (21). Previous z-scan studies established that the axial distribution of EGFP-HRas in the cytoplasm and at the PM membranes was successfully modeled by the $\delta S \delta$ layer geometry $(21,22)$. Thus, DC z-scans from cells co-expressing mCherry and EGFP-HRas were modeled by the $\left(\mathrm{S}^{\mathrm{CH}}-\delta S \delta\right)^{\mathrm{G}}$ geometry (Eq. 17). A fit of a representative $z$-scan attained good quality of fit with $x_{v}{ }^{2}=1.2$ and flat residuals. Repeated z-scans in the cytoplasm of multiple cells co-expressing EGFP-HRas and mCherry (Fig. 1I) determined $\langle\Delta>=-2.6 \pm 5.4 \mathrm{~nm}$ (SEM, $n=30$ ), which was within $0.5 \mathrm{SD}$ of the expected value of $\Delta=0$. This result indicated that the location of the cytoplasm slab edge, as determined by fitting the soluble mCherry species to the S model, coincided with the location of the PM, as determined by fitting the EGFP-HRas species to the $\delta S \delta$ model. 
Next, the axial cytoplasmic distribution of APOBEC 3G (A3G) labeled with EGFP and co-expressed

with mCherry (Fig. 1J) was examined. A representative z-scan acquired in the cytoplasm of a HeLa cell showed a single broad peak for both mCherry and A3G-EGFP (Fig. 1K). Since the trace shape was suggestive of a slab geometry, both species were fit to slab models $\left(\mathrm{S}^{\mathrm{CH}} \mathrm{S}^{\mathrm{G}}\right.$, Eq. 17). This fit provided a good description of the DC z-scan intensity profiles with $X v^{2}=1.3$ and flat residuals (Fig. 1K). Interestingly, this fit identified non-zero values for the displacements at the top and bottom PM $\left(\Delta^{B}=184 \mathrm{~nm}\right.$ and $\Delta^{T}=167$ nm). Repeated z-scan acquisitions at the same location in this cell determined $\overline{\Delta^{B}}=180 \pm 6 \mathrm{~nm}$ and $\overline{\Delta^{T}}=$ $162 \pm 12 \mathrm{~nm}$ (SEM, $n=12$ ). This surprising observation suggests that the thickness of the soluble A3GEGFP layer is less than the thickness of the mCherry layer, unlike the results obtained for EGFP and mCherry (Fig. 1D), which had equal lengths $(\Delta=0)$ for both soluble proteins. Repeated z-scan acquisitions in multiple HeLa cells (Fig. $1 \mathrm{~L}$ ) found $<\Delta>$ HeLa $=155 \pm 10 \mathrm{~nm}$ (SEM, $n=64$ ), indicating that $A 3 G$ does not occupy the entire cytoplasmic volume. To further validate this observation, DC z-scan measurements were collected using a different cell line (U2OS), which resulted in $<\Delta>\mathrm{U} 20 \mathrm{OS}=110 \pm 9 \mathrm{~nm}(\mathrm{SEM}, n=32)$.

Intriguingly, this result implies that cytoplasmic A3G-EGFP was excluded from regions adjacent to the PM with apparent thickness of $100-200 \mathrm{~nm}$. Given the magnitude of this value and its differential value in U2OS and HeLa cell lines, further experiments were conducted to test whether A3G-EGFP might be partially excluded from the dense meshwork of the actin cortex. To do this, HeLa cells were treated with ethanol (see Materials and Methods), which disrupts actin assemblies and leads to the formation of large plasma membrane blebs (23) (Fig. 2A) that lack an intact actin cortex (24-26). A representative z-scan performed near the center of these blebs showed good quality of fit to a $\mathrm{S}^{\mathrm{CH}}-\mathrm{S}^{\mathrm{G}}$ model (Eq. 17 and Fig. 2C). Repeated z-scans in blebs from HeLa cells co-expressing A3G-EGFP and mCherry determined $<\Delta>=31 \pm$ $10 \mathrm{~nm}$ (SEM, $n=14$ ), which differs from the results obtained in untreated HeLa cells by 8.8 SD. The distribution of $\Delta$ in blebs and in untreated cells are markedly different (Fig. 2D). The strong reduction in $\Delta$ found in blebs supports the conclusion that A3G-EGFP concentration is depleted from the vicinity of the PM by the presence of the actin cortex. To reflect these results $\Delta$ is also referred to as the effective depletion length.

Given that dependence of cortical architecture on the cell type has been observed (27-29), the differences in effective depletion length observed between HeLa and U2OS cells might reflect natural differences in cortex thickness between these cell types. To investigate this, direct measurements of the cortex thickness were performed using distinct fluorescent colors to mark the actin cortex and the plasma 
membrane. A previous study used this approach to estimate the actin cortex thickness from the displacement between intensity peaks corresponding to the actin and the PM marker in confocal images (30). In the present study two-photon DC z-scan was employed instead of confocal imaging to determine the cortex thickness from the intensity profiles of EGFP-HRas (which binds to the PM) and Lifeact-mApple (which binds to actin and marks the cortex) (31) by fitting to on a quantitative model of the entire intensity traces (Fig. 3A). The axial concentration profile of both labels is depicted in Fig. 3B, which shows an enrichment of EGFPHRas and Lifeact-mApple at the PM and within the cortex, respectively. A small amount of both fluorescent proteins is also present in the cytoplasm. Based on this profile, EGFP-HRas is again modeled by the $\delta S \delta$ geometry. The distance separating the two HRas $\delta$ layers represents the cell thickness $L_{\text {cell }}$.

The concentration profile of Lifeact-mApple implies a wide cytoplasmic slab of length $L_{\text {cyto }}$ separating two thin slabs representing the cortex at the bottom and the top membrane with thickness $L_{C}^{B}$ and $L_{C}^{T}$ (Fig. 3B), giving rise to an $\mathrm{SSS}^{\mathrm{CH}}$ (triple S-layer) geometry. Choosing a uniform slab with a sharp transition between the cortical and cytoplasmic spaces to model the cortex has been previously used in (30) where it is estimated that even a substantial non-uniformity of the cortical density leads to only a small change in the estimated cortex thickness. Because the expected slab length $L_{c}$ of the cortex is smaller than $500 \mathrm{~nm}$, its length cannot be directly identified in single-color (SC) z-scan experiments. Such thin slabs are experimentally indistinguishable from a $\delta$ layer (32). However, the centroid position of the thin cortical layer is expected to coincide with the midpoint of the slab as depicted in Fig. 3B. Given this, modeling the LifeactmApple species as a $\delta S \delta^{\mathrm{CH}}$ geometry can identify the cortex centroid from the fitted location of each $\delta$ layer. Combined with the EGFP-HRas species, this DC z-scan fit $\left(\delta S \delta^{C H}-\delta S \delta^{G}\right)$ identifies the locations of the $\delta$ layers corresponding to the cortex centroid and the PM as well as their displacement $\Delta$. The distance $|\Delta|$ separating both $\delta$ layers is half of the actin cortex thickness, $|\Delta|=L_{C} / 2$ (Fig. 3B).

The relation $|\Delta|=L_{C} / 2$ was verified by computer modeling of the DC z-scan intensity profile of the SSS ${ }^{C H}-\delta S \delta^{G}$ geometry (Fig. 3B), while systematically varying the cortical thickness $L_{C, \text {,in }}$, as well as the cell thickness and fluorescence intensities of HRas and Lifeact (see Materials and Methods). These simulated scans were fit to a $\delta S \delta^{C H}-\delta S \delta^{G}$ model to determine the locations of each $\delta$ layer. The difference $\Delta$ between the fitted cortical $\delta$ layer and the PM $\delta$ layer was computed for each fit and the averaged $\Delta$ recovered from fits to simulated DC z-scans were plotted as a function of the cortical thickness $L c$,in (Fig. S1). The data 
153 confirm that the relation $|\Delta|=L_{c} / 2$ is accurate to within $10 \mathrm{~nm}$ for cortex thicknesses up to $200 \mathrm{~nm}$. This 154 result demonstrates that this approach is experimentally accurate and produces negligible error.

Repeated DC z-scans were then collected in cells co-expressing EGFP-HRas and Lifeact-mApple proteins in order to determine cortical thickness. A representative $\mathrm{DC}$ z-scan and fit to the $\delta S \delta^{\mathrm{CH}}-\delta S \delta^{\mathrm{G}}$ model (Fig. 3C) for both proteins is shown in Fig. 3D. Data taken from multiple cells resulted in absolute values $|<\Delta>|$ UrOS $=63 \pm 8 \mathrm{~nm}(\mathrm{SEM}, n=7)$ and $|<\Delta>|_{\text {HeLa }}=100 \pm 13 \mathrm{~nm}(\mathrm{SEM}, n=15)$. These values correspond to uniform cortex thicknesses of $126 \pm 16 \mathrm{~nm}$ for U2OS cells and $200 \pm 24 \mathrm{~nm}$ for HeLa cells (Fig. 3E). cell lines, $<\Delta>$ in HeLa and U2OS cells were plotted for comparison (Fig. 3F). DC z-scans were performed

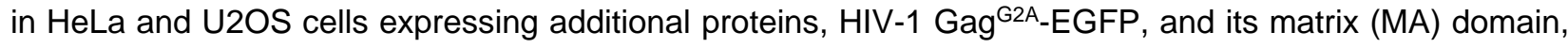
$M^{\text {G2A }}$-EGFP (33) with mCherry again as a reference marker of the cytoplasmic volume for each instance. A cell-line dependent $<\Delta>$ was observed for both proteins (Fig. S2), and their $<\Delta>$ values were added to Fig. 3F. Interestingly, all 3 data points cluster near a line of slope 0.66 , indicating that, although the magnitude of the depletion effect varies for each protein, it is systematically scaled by $\sim 66 \%$ between these two cell lines. This same ratio corresponds well to the cortex thickness ratio estimated by DC z-scan between HeLa and U2OS cells $(0.66 \pm 0.11$, shaded area in Fig. 3F), suggesting that the cell-type dependent differences in $<\Delta>$ are due to differences in their cortical actin thicknesses. This result further implicates the actin cortex as a central driver of the observed depletion effect near the PM.

All 3 proteins in Fig. 3F are known to be RNA-interacting proteins: HIV-1 Gag ${ }^{\mathrm{G} 2 \mathrm{~A}}$ binds specifically and non-specifically to RNA sequences through basic residues and two zinc fingers in its nucleocapsid (NC) domain (34-36), while the MA domain of Gag separately interacts with RNA via a stretch of basic residues in its highly basic region (HBR) (37-39) and has preferential tRNA binding affinity $(40,41)$. A3G interacts with RNA primarily through its $\mathrm{N}$-terminal domain zinc finger $(42,43)$. A3G-RNA binding is required for packaging of $A 3 G$ into retroviral particles (43-46) and is critical to its antiretroviral activity $(43,44)$. To test whether the depletion effect is specific to RNA-binding proteins, measurements with two EGFP-labeled proteins that lack a known RNA binding function were conducted. In particular, DC z-scans of HeLa cells coexpressing mCherry with the GABA-A receptor associated protein fused to EGFP (GABARAP-EGFP), and mCherry with a tandem hexamer of the Venus fluorescent protein, Venus 6 were collected. The average $<\Delta>$ value of both proteins was found to be $<10 \mathrm{~nm}$ (Fig. 4A) and therefore treated as consistent with zero. The 
183 previously presented depletion lengths in HeLa cells from Fig. 3D were added to Fig. 4A to highlight the 184 contrast with the RNA-binding proteins. Since RNA binding appeared to be important for observing depletion, DC z-scans were performed with the mutant A3GC97A-EGFP, which has impaired RNA binding due to 186 mutation of the $\mathrm{N}$-terminal zinc finger (44). These measurements revealed a large reduction in $\langle\Delta\rangle$ 187 compared to that of wildtype A3G-EGFP (Fig. 4A). Lastly, the $<\Delta>$ value was measured in mCherry expressing HeLa cells that were stained with the small molecule RNA binding dye, SYTO RNASelect. These measurements revealed that the labeled cellular RNA had a significant degree of depletion adjacent to the PM (Fig. 4A).

Based on these results, it was hypothesized that the exclusion of large RNA species from the dense cortical meshwork may be responsible for the observed PM adjacent depletion of RNA binding proteins (Fig. 4B). The average pore diameter of the actin cortex has been reported to range between 50 to $200 \mathrm{~nm}$ (28), depending on cell type. These values are only slightly larger than or overlap with the size of large RNAs (6, 7). Thus, a partial partitioning of RNAs from the interior of the cortex could be driven by excluded volume interactions. In a simple model of the cortical partitioning (Fig. 4C), the ratio of the concentration $c_{c}$ in the cortical layer to the concentration $c_{\text {cyto }}$ in the cytoplasm defines the partition coefficient $R_{C}=c_{C} / c_{\text {cyto }}$. This partitioning of the protein concentration between the cytoplasmic and cortical spaces introduces a SSS concentration profile for the EGFP-labeled RNA-binding protein (Fig. 4C), which consists of two thin cortical slabs of length $L_{C}^{B}$ and $L_{C}^{T}$ separated by a thick cytoplasmic slab of length $L_{c y t o}$ with a higher protein concentration. As in the case of the Lifeact measurements (Fig. 3), the cortical layer is too narrow to be directly resolved by z-scan analysis. However, since the concentration within the cortex layer is reduced (Fig. 4C), the z-scan intensity profile can be effectively approximated by a single slab layer with a width that slightly exceeds the cytoplasmic thickness $L_{\text {cyto }}$ (Fig. 4D). Consequently, the apparent depletion lengths $\Delta^{B}$ and $\Delta^{T}$ will be less than the corresponding cortex thickness $L_{C}^{B}$ and $L_{C}^{T}$ in a manner that depends on $R_{c}$.

To quantitatively analyze how $\Delta$ recovered from DC z-scans is related to $R_{C}$ and $L_{C}$, simulation of DC z-scan traces for an $\mathrm{SSS}^{\mathrm{G}}-\mathrm{S}^{\mathrm{CH}}$ geometry was performed (see Materials and Methods). These simulated traces were fit to the $S^{C_{-}} S^{G} D C$ z-scan model (Fig. 4D). The cortex length $L_{C}$ and partition ratio $R_{C}$ (Fig. 4C) were varied for each simulation and the averaged $\Delta$ recovered from the fits was plotted (Fig. $4 \mathrm{E}$, diamonds). The results of these simulations indicate a nearly linear relationship between $\Delta$ and $R_{c}$ for a given $L_{c}$. This linear relationship can also be derived from preserving the integrated area $\Lambda$ under the z-scan intensity 
212 profile between the $S S S^{G}$ model and the fitted $S^{G}$ model. Since $\Lambda$ and the axially integrated concentration $A$ 213 are directly proportional (Eq. 11), the concentration areas of both models (Fig. 4C and D) must match. In

214 particular, the cortical concentration area $A_{C}^{i}=L_{C}^{i} \cdot c_{C}$ (Fig. 4C) has to be equal to the added extra area

$215 A_{x}^{i}=L_{C}^{i} \cdot R_{C} c_{\text {cyto }}$ (Fig. 4D) at both the top $(\mathrm{i}=\mathrm{T})$ and bottom $(\mathrm{i}=\mathrm{B})$ of the cell, which predicts an apparent 216 depletion length $\Delta$ (Fig. 4D) of

218 where the superscript has been omitted. This model (Fig. 4E, solid lines) is in excellent agreement with the 219 results from the simulations. Eq. 1 was used to estimate the partition coefficient of proteins from their measured depletion lengths (Fig. 3D) using the experimentally determined cortex thicknesses $L_{c} . R_{c}=0.76$ $221 \pm 0.02,0.35 \pm 0.04$, and $0.24 \pm 0.05$ for MA ${ }^{\mathrm{G} 2 \mathrm{~A}}$-EGFP, Gag ${ }^{\mathrm{G} 2 \mathrm{~A}}$-EGFP, and A3G-EGFP, respectively were 222 determined from HeLa cells. Similar values $(0.76 \pm 0.02,0.41 \pm 0.02$, and $0.17 \pm 0.07)$ were obtained from 223 the measurements in U2OS cells.

Because these estimates of the partition coefficient $R_{C}$ rely on the measured displacement $\Delta$, which is a new method, a second approach was developed to estimate $R_{c}$ independently of $\Delta$ by using DC z-scans in very thin cytoplasmic regions. The partial exclusion of fluorescently labeled protein from the cortex reduces the fluorescence area $\Lambda^{G}$ as compared to the area $\Lambda_{r e f}^{G}$ corresponding to the hypothetical case of no exclusion. The ratio of these two areas is denoted as the axially averaged partition coefficient,

$$
\bar{R}_{z}=\frac{\Lambda^{G}}{\Lambda_{\mathrm{ref}}^{G}}=\frac{A^{G}}{A_{\mathrm{ref}}^{G}}=\frac{L_{c y t o} c_{c y t o}+2 L_{C} R_{C} c_{c y t o}}{L_{\text {cell }} c_{c y t o}},
$$

where the proportionality between $\Lambda$ and $A$ (Eq. 11) was used to express the ratio in terms of the integrated concentration areas. Since the cell thickness is defined by (Fig. 3B),

$$
L_{\text {cell }}=L_{\text {cyto }}+L_{C}^{T}+L_{C}^{B}=L_{c y t o}+2 L_{C}
$$

233 where $L_{C}=\left(L_{C}^{T}+L_{C}^{B}\right) / 2$ defines the average cortex thickness, the axially averaged partition coefficient 234 simplifies to

$$
\bar{R}_{z}=1+\left(R_{C}-1\right) \frac{2 L_{C}}{L_{\text {cell }}}
$$


236 This equation predicts $\bar{R}_{z} \rightarrow 1$ for very thick sections $\left(L_{c y t o} \gg L_{C}\right)$. As the cell thickness $L_{\text {cell }}$ decreases,

237 the value of $\bar{R}_{z}$ drops. It reaches a limiting value of $\bar{R}_{z}=R_{C}$ for very thin cell section composed entirely of 238 cortex $L_{\text {cell }}=2 L_{C}$. Thus, DC z-scans in thin regions near the cell perimeter combined with DC z-scans in 239 thick regions of the same cells allow direct estimation of the partition coefficient.

241 the average cortical thickness in a given cell type, very thin cellular regions were found where the realized 242 cell thickness is less than this value, $L_{\text {cell }}<2 L_{C}$. This implies that in these regions of the cell the local cortex 243 thickness is less than $L_{C}$. Assuming that these thin sections are composed entirely of cortex, Eq. 4 is 244 generalized to,

$$
\bar{R}_{z}=\left\{\begin{array}{ccc}
R_{C} & \text { if } & L_{\text {cell }}<2 L_{C} \\
1+\left(R_{C}-1\right) \frac{2 L_{C}}{L_{\text {cell }}} & \text { if } & L_{\text {cell }}>2 L_{C}
\end{array} .\right.
$$

As shown in Fig. 5A \& B, cytoplasmic DC z-scans in thick and thin regions of a cell co-expressing

EGFP and mCherry indicate that the relative concentrations of the EGFP and mCherry species are identical regardless of cytoplasm thickness. In contrast, cytoplasmic DC z-scans in thick and thin regions of a cell coexpressing HIV-1 Gag ${ }^{\mathrm{G} 2 \mathrm{~A}}$-EGFP and mCherry show that the relative intensity of Gag ${ }^{\mathrm{G} 2 \mathrm{~A}}$-EGFP is sharply reduced in thin regions (Fig. 5C \& D), demonstrating that the depletion effect is reflected in the decrease of

251 the integrated fluorescence area $\Lambda^{G}$ as a function of thickness. To quantify the effect and utilize Eq. 2, an 252 estimate was needed for the reference area $\Lambda_{\text {ref }}^{G}=L_{\text {cell }} g_{\text {cyto }}^{G}$, where $g_{\text {cyto }}^{G}=c_{\text {cyto }}^{G} \eta$ (Eqs. 11 and 2). The cell thickness $L_{\text {cell }}$ was determined from the mCherry signal in the red detection channel as described in the Material and Methods, while the amplitude $g_{\text {cyto }}^{G}$ was estimated from a scan through a thick cell section $\left(L_{c e l l}\right.$

$>1.5 \mu \mathrm{m}$ ) where $g_{\text {cyto }}^{G}$ can be reliably extracted from fitting the DC z-scan traces. Repeated z-scans were performed within the same cell covering thick and thin regions (Fig. 5E) to graph $\bar{R}_{z}=\Lambda^{G} / \Lambda_{\text {ref }}^{G}$ versus $L_{\text {cell. }}$.

As expected, $\bar{R}_{z}$ is approximately one for a HeLa cell coexpressing mCherry and EGFP (Fig. 5F), which 
scan estimates of $\bar{R}_{z}$ for Gag ${ }^{\mathrm{G} 2 \mathrm{~A}-\mathrm{EGFP}}$ for a HeLa cell (magenta diamonds) and a U2OS cell (blue 260 diamonds) exhibit a decrease at thin cell sections (Fig. 5F). The HeLa cell data in Fig. 5F closely resemble 261 the simple model (Eq. 5), and cluster near 0.2 for $L_{\text {cell }}<L_{C}$, suggesting a cortical partition coefficient near 2620.2 in this cell line. The data from U2OS cells appears to deviate from (Eq. 5) in thin cell sections, which may reflect the presence of distinct peripheral F-actin structures in this cell line as discussed later.

The cortical partitioning effect documented here supports a model where protein depletion within the cortex is driven by protein association into ribonucleoprotein complexes that are much larger than the typical protein. In such a case, the large $\langle\Delta\rangle$ values should be correlated with substantially slowed cytoplasmic diffusion. FCS measurements were performed in HeLa cells in order to estimate the effective diffusion coefficient $D_{\text {eff }}$ of labeled proteins in the cytoplasm (see Materials and Methods). In Fig. $6 \mathrm{~A}<\Delta>$

values from Fig. 4 were plotted versus the median $D_{\text {eff }}$ that was obtained from FCS measurements. Two

other members of the APOBEC3 protein family, A3A-EGFP and A3C-EGFP, which have differing antiretroviral activities (47-49), were also measured and added to Fig. 6A. These data show that large $\langle\Delta\rangle$ values are associated with remarkably low cytoplasmic mobility. This relationship is summarized by the cartoons in Fig. 6 B\&C. In order to model the data in Fig. 6A, the cortical partitioning was described as arising from excluded volume interactions of particles that are allowed to enter spherical voids (50-52),

$$
R_{C}=\left(1-r_{p} / r_{v}\right)^{3}
$$

where $r_{p}$ is the particle radius, and $r_{v}$ is the void radius. A hindered cytoplasmic diffusion coefficient $D_{\text {eff }}$ was modeled as arising from a size-dependent viscosity imposed by macromolecular crowding within the cytoplasm $(53,54)$ according to

$$
\frac{D_{\text {eff }}}{D_{0}}=\exp \left[-\left(\frac{r_{\text {eff }}}{\xi}\right)^{a}\right] \text {, }
$$
particles, $a$ is a scaling exponent of order 1 , and $\xi$ is a length interpreted as the characteristic distance between points of entanglement within the crowded solution. Along with equation 1 , setting $r_{\text {eff }}=r_{p}$ in the above yields a relation between $D_{\text {eff }}$ and $\Delta$, 


$$
\Delta=L_{c}\left[1-\left(1-\xi / r_{v} \ln \left(D_{0} / D_{\text {eff }}\right)^{1 / a}\right)^{3}\right]
$$

This curve is plotted as the magenta line in Fig. $6 \mathrm{~A}$ using values $\underline{\underline{L}} \underline{c}=170 \mathrm{~nm}, D_{0}$ equal to $D_{\text {eff }}$ measured for EGFP $\left(27.5 \mu \mathrm{m}^{2} \mathrm{~s}^{-1}\right), r_{v}=60 \mathrm{~nm}, \xi=4 \mathrm{~nm}$ and $a=0.7$. The values for $\xi$ and $a$ are within the range estimated by (53) for HeLa cells. $r_{v}$ is similar to published estimates of the cortical mesh size (28).

Cytoplasmic crowding in cell associated PM blebs may differ from crowding within undisturbed cytoplasm due at least in part to the lack of a cytoskeletal mesh in the bleb interior. Thus, the mobility of A3G-EGFP in HeLa cell blebs was investigated. FCS measurements (Fig. 6A, open circle) showed an order of magnitude increase in $D_{\text {eff }}$ compared to $A 3 G$ in the cytoplasm of untreated HeLa cells. This result supports our choice to model cytoplasmic diffusion of RNP complexes as arising from a size-dependent viscosity (Eq. 717). The value from blebs is plotted as an open circle in order to emphasize that the model parameters (especially $r_{v}$ and $\xi$ ) that describe the rest of the data most likely do not describe data from blebs.

It was recently reported that the cytoplasmic diffusion coefficient of fluorescent proteins depended on their net charge, with positively charged proteins diffusing slowly. It was suggested that this slow diffusion was due to transient interactions with large, negatively charged cell components such as RNA and the cytoskeleton (55). An examination was performed to determine whether the $D_{\text {eff }}$ values in Fig. $6 \mathrm{~A}$ might be explained by the net charge of each protein-FP fusion. As shown in Fig. S3, the mobility of the proteins in Fig. $6 \mathrm{~A}$ was only weakly correlated with net charge. Thus, the mobility of these proteins in living cells is likely to be better understood as arising predominantly from their RNA binding function.

\section{Discussion}

In this study, DC z-scan microscopy measurements of the fluorescence intensity profiles of green and red labeled proteins provided the foundation for identifying the cortical partitioning effect and allowed its quantitative description through the effective depletion length $\Delta$. Implementation of the DC z-scan measurements using two-photon microscopy eliminates systematic biases, such as chromatic aberration, from the collected data. DC z-scan permitted precise quantification of the relative localizations of fluorescently labeled proteins. These measurements indicated an apparent restriction in the cytoplasmic volume of RNA binding proteins (e.g., $A 3 G$ and $G a g^{G 2 A}$ ). The partitioning effect was markedly reduced in 
311 ethanol induced cell blebs, which largely lack a cortex (24-26) and was observed to be cell-type dependent 312 in a manner that correlated with DC z-scan estimates of cortex thickness in adherent interphase HeLa and 313 U2OS cells. The depletion effect is interpreted as being a partitioning, or partial exclusion, of these proteins 314 from the volume of the actin cortex. Furthermore, $\Delta$ values measured for proteins with and without RNA315 binding function suggest that the cortical partitioning is driven by association into RNP complexes and is not 316 strongly associated with protein molecular mass or net charge. This conclusion is supported by 317 measurements of the diffusion coefficient, which shows that a marked decrease in mobility is associated 318 with proteins that have the largest values of $\Delta$.

In vitro and in vivo (56) observations of actin assemblies suggest that gel-like properties are nearly ubiquitous, including for the actin cortex specifically (57). The cortical partitioning effect may be analogous to solute partitioning from the interior of gels, a well-known chemical phenomenon $(58,59)$. Furthermore, the subcellular heterogeneity driven by the dense cortical environment is reminiscent of those molecular crowding effects which have been reported to drive association or phase separations in cells $(60,61)$. This suggests that the crowded environment within gels may drive biophysical phenomena similar to, but distinct from those associated with molecular crowding in the cytoplasm. The observation of cortical partitioning described in this report builds on earlier work that found strong exclusion of labeled colloidal particles with radii exceeding $\sim 10 \mathrm{~nm}$ from distal regions of mammalian cell cytoplasm, and regions near the centrosome $(15,62)$. Subsequent studies documented that the distal excluding regions were associated with a dense cytoskeletal mesh and were thinner than non-excluding regions (62). These and other observations (50) have suggested that this partitioning was driven by the cytoskeletal meshwork.

The cortical partitioning of RNPs described in the present study also leads to strong exclusion from distal regions of the cytoplasm (Fig. 5). However, unlike previous studies, which linked partitioning to the actin cytoskeleton in general, our observations identified the actin cortex as the causative agent of partitioning. Differentiation between the cortical space and the bulk cytoplasm was not previously achieved and probably reflects experimental limitations in these earlier studies, which are overcome by the use of DC z-scan method employed here. Thus, the cortical partitioning of RNPs and the distal exclusion of microinjected particles documented previously $(15,63)$ likely are manifestations of the same physical phenomenon, namely the size-dependent partitioning of particles from the actin cortex.

The measurement of the actin cortex thickness, $L_{C}$, in HeLa $(\sim 200 \mathrm{~nm})$ and U2OS ( 130 nm) cells in our study represents a novel application of the DC z-scan technique. This application was inspired by a conceptually similar approach based on confocal microscopy that was used to estimate a cortex thickness 
of $190 \mathrm{~nm}$ in mitotic HeLa cells (30) and of $300-400 \mathrm{~nm}$ in interphase HeLa cells with imposed spherical shapes (29). Interestingly, DC z-scan identified a cortical thickness $\leq 200 \mathrm{~nm}$ for adherent interphase HeLa and U2OS. This is consistent with literature reports which indicate that cortical architecture is strongly associated with cell morphology $(27,29)$. In particular, cell adhesion and cell spreading on the glass coverslip can result in a thinning of the actin cortex $(27,64)$.

DC z-scan measurement of the axially averaged partition coefficient $\bar{R}_{z}$ (Fig. 5E) provided further

in increasingly thinner cell sections. The value of $\bar{R}_{z}$ in very thin cell sections provides a direct estimate of the cortical partition coefficient $R_{c}$, assuming that these thin sections are composed entirely of the actin cortical mesh. This $R_{c}$ value can in turn provide an additional estimate of cortex thickness that does not rely on DC z-scans of cortical Lifeact. Utilizing Eq. 1, the $R_{C} \sim 0.2$ measured for HIV-1 Gag ${ }^{\mathrm{G} 2 \mathrm{~A}}$-EGFP in thin sections of HeLa cells would suggest $L_{C} \sim 170 \mathrm{~nm}$ given $\langle\Delta\rangle=135 \pm 8 \mathrm{~nm}$ observed for HIV-1 Gag ${ }^{\text {G2A }}$-EGFP in thick cytoplasmic DC z-scans in this same cell line. This value of $L_{c}$ is within $1.3 \mathrm{SD}$ of the value estimated from DC z-scans of Lifeact. The agreement between these two different DC z-scan approaches provides further validation of the DC z-scan technique and demonstrates its utility for interrogating biophysical properties of the bulk actin cortex.

Interestingly, DC z-scan estimates of $\bar{R}_{z}$ in U2OS cells exhibited more scatter than observed in HeLa cells, particularly in thin cell sections. This may reflect cell-type differences in F-actin assemblies, in particular large structures such as lamellar actin sheets which may be present near the cell periphery. In electron micrographs of F-actin, these lamellae appear distinct from cortical actin fibers $(27,65)$. Their extensive presence may invalidate the use of Eq. 5 near the periphery of U2OS cells. Future work is needed to investigate whether distinct types of actin structures modulate the cortical partitioning and whether this effect is cell-type and -function dependent.

The strong correlation between the ability of a protein to bind RNA and measured values of $<\Delta>$ (Fig. 4A) suggests that many RNA-protein complexes are too large to efficiently enter the dense actin cortical meshwork. The cortical partitioning of RNA-binding proteins measured by DC z-scan is thus interpreted as arising from an underlying partitioning associated with the large RNA species with which these proteins associate. In this framework, DC z-scans of RNA interacting proteins indirectly probe the spatial distribution of cellular RNAs. More directly, when cellular RNA was labeled with an RNA-binding fluorescent dye, DC z- 
scan measurements of $\left\langle\Delta>\right.$ revealed that the RNA itself is partitioned from the cortex with $R_{C}$ in the range $0.35-0.2$ (given $L_{C} 170-200 \mathrm{~nm}$ ). This $R_{C}$ value is interpreted as representing the average $R_{C}$ of a broad spectrum of differently sized cellular RNAs (i.e., mRNAs, tRNAs, etc) and RNP complexes (e.g., ribosomes) with different degrees of cortical penetration. Similarly, the $\langle\Delta\rangle$ values for fluorescently labeled proteins likely represent the average $R c$ of a heterogenous mix of differently sized RNA complexes to which each protein preferentially binds. Alternatively, it is possible that different $\langle\Delta\rangle$ values represent differing levels of RNA-bound and -unbound protein, where the unbound state is identified as the non-partitioned fraction. However, the unbound protein population is expected to be negligible in many cases. This follows from the high concentration of protein-RNA binding sites in cell cytoplasm $(66,67)$, which is estimated to greatly exceed both the protein-RNA affinities (likely tens of nanomolar for Gag and A3G binding to single strand RNAs $(42,68,69))$ and the protein concentrations $(\sim 1 \mu \mathrm{M})$ in DC z-scan measurements. The potential for protein-RNA binding equilibria or selective protein-RNA binding to influence the cortical partitioning effect may be explored with further studies utilizing DC z-scan.

FCS measurements were acquired in order to characterize protein mobility in the cytoplasm. Given that these data were acquired with the PSF focused near the middle of thick $(>1.5 \mu \mathrm{m})$ cytoplasmic sections, the experimental estimates mainly report the mobility within the cytoplasm and not the mobility within the actin cortex. The diffusion time of RNA binding proteins revealed that large $<\Delta>$ values were associated with very low mobility (Fig. 6A), as expected for proteins that are present in large complexes. Slow diffusion of large sized objects in the cytoplasm has been previously reported $(14,17,70,71)$, including mRNAs (72). Previous studies have suggested that this low cytoplasmic mobility may reflect a hindrance created by cytoskeletal elements $(15,16,18,19,62,73,74)$ or by cytoplasmic crowding created due to ribosomes, organelles and proteins $(17,70,75-77)$. Although the precise determinants for cytoplasmic mobility have not been fully characterized, existing literature provides extensive evidence that the cytoplasm cannot be described as a simple fluid $(12,53,75,78)$, and it is generally agreed that particles in the range of $10-$ $100 \mathrm{~nm}$ show a size-dependent drop in cytoplasmic mobility much greater than that predicted by the StokesEinstein relation $(14,17,18,70)$. In this study, a marked increase in A3G-EGFP mobility in cell-associated blebs (Fig. 6A) was observed. This suggests a role for the cytoskeleton in the mobility of large macromolecular complexes.

To capture the size dependence of RNP diffusion, an empirical hydrodynamic scaling model (Eq. 7 ) was used. This and similar models have been previously employed to describe diffusion in polymer solutions and in living cells $(53,54)$. This model led to good agreement with our experimental data (Fig. $6 \mathrm{~A})$ 
402 when combined with a relatively simple description of the size dependence of cortical partitioning (50-52)

403 (Eq. 6). This result demonstrates the feasibility of a quantitative description of the actin cortical partitioning 404 effect. The choice to model the partition coefficient as arising from the excluded volume due to spherical 405 cavity (Eq. 6) was driven primarily by the simplicity of this approach. The excluded volume due to a mesh of 406 randomly oriented fibers, which may provide a more realistic model of cortical actin, is expected to give 407 similar results to Eq. $6(50)$ at the expense of an additional free parameter.

\section{Materials and Methods}

\section{Cell Culture and Plasmids}

HeLa and U2OS (Numbers CCL-2 and HTB-96, American Type Culture Collection, Manassas, VA) cells were cultured in Dulbecco's modified Eagle's medium supplemented with 10\% FBS (HyClone Laboratories, Logan, UT) at $37^{\circ} \mathrm{C}$ and $5 \% \mathrm{CO}_{2}$. Cells were plated at $\sim 30 \%$ confluency into 8-well chambered slides $\sim 24 \mathrm{~h}$ prior to measurement and transfected with vectors as indicated in the text $\sim 16 \mathrm{~h}$ prior to measurement using 
432 GenJet transfection reagent (SignaGen Laboratories, Fredrick, MD) according to the manufacturer's 433 instructions. The cell growth medium was replaced with phosphate buffered saline immediately prior to 434 measurement. All measurements were performed at room temperature.

435 HIV-1 Gag (84) was ligated into pEGFP-N1 (Clontech, Takara Bio, Mountain View, CA) to generate the HIV4361 Gag-EGFP vector. HIV-1 Gag ${ }^{\text {G2A }}$-EGFP was made using the QuikChange XL Site-Directed Mutagenesis 437 Kit (Stratagene, La Jolla, CA). Venus6 was a gift from Steven Vogel (85) (Addgene plasmid \# 27813; 438 http://n2t.net/addgene:27813; RRID: Addgene_27813). mApple-Lifeact-7 (denoted Lifeact-mApple here) 439 was a gift from Michael Davidson (Addgene plasmid \# 54747; http://n2t.net/addgene:54747; RRID: 440 Addgene_54747). All other vectors have been previously described: A3G-EGFP, A3GC97A-EGFP, A3C441 EGFP, A3A-EGFP (44); HIV-1 MAG2A-EGFP (20); mCherry (86); EGFP-HRas (21); mCherry-RXR (87); 442 GABARAP-EGFP (88).

\section{Instrumentation}

DC z-scans and fluorescence fluctuation spectroscopy (FFS) data were acquired on a Zeiss Axiovert 200 microscope modified for 2-photon excitation (20). Excitation light was provided by a femtosecond pulsed Ti:S laser (MaiTai or Tsunami models, Spectra Physics, Santa Clara, CA) operated at $1000 \mathrm{~nm}$. This light was focused to a diffraction limited point by a water immersion objective (C-Apochromat, Zeiss, Oberkochen, Germany) with numerical aperture 1.2 and correction collar set according to the glass thickness of the sample holder. Emission light was collected by the same objective and separated from the excitation light by a dichroic filter (740DCSPXR, Chroma Technology, Bellows Falls, VT), then split into red and green detection channels by a low-pass dichroic (FF580-FDi01, Semrock, Rochester, NY). A 515/50nm band pass filter placed before the green channel detector eliminated Fresnel reflected mCherry emission light from the green channel. Emission light in each channel was detected by single photon counting hybrid photomultipliers (HPM-100-40, Becker and Hickl, Berlin, Germany), recorded by hardware (FastFLIM, ISS Inc., Champaign, IL or DPC-230, Becker and Hickl, Berlin, Germany), and saved for later analysis using routines written in IDL 8.6 (Harris Geospatial Solutions, Broomfield, CO).

Axial z-scans utilized a piezo stage (PZ2000, ASI, Eugene OR) driven by a voltage waveform from an arbitrary waveform generator (model 33250A, Agilent Technologies, Santa Clara, CA) to move the sample vertically across the two-photon point spread function (PSF). All z-scans were acquired by driving the piezo stage with a triangle waveform with a $10 \mathrm{~s}$ period and peak-to-peak displacement corresponding to $24 \mu \mathrm{m}$. 
462 Each period contained two z-scans: the first z-scan passed upward through the cell and the second passed downward through the cell. Each z-scan covered a $24 \mu \mathrm{m}$ range.

XY scanning of the PSF for acquisition of two-photon images was provided by a galvanometer scan

head (Yanus IV, FEI Deutschland GmbH, Planegg, Germany) aligned in the two-photon excitation path. XY positioning was driven by a DAC card (3-axis, ISS Inc., Champaign, IL) controlled by SimFCS software (version 3; G-SOFT Inc., Champaign, IL). All z-scans were acquired within $24 \mu \mathrm{m}$ of the XY scan field center.

\section{Z-scan modeling}

The single-channel (SC) z-scan fluorescence intensity profile $F(z)$ is determined by the convolution of the

axial concentration profile $c(z)$ of the fluorescently-tagged protein and the radially integrated point spread function (RIPSF) of the two-photon instrument as modeled in $(22,32)$,

$$
F(z)=\lambda(c * \operatorname{RIPSF})(z)
$$

where $\lambda$ is the brightness of the fluorescence tag. Evaluation of Eq. 9 for different concentration profiles $c(z)$ gives rise to an alternative formulation with a fluorescence amplitude $f$ and a function $\mathrm{v}(z)$ describing the shape of the concentration profile (32),

$$
F(z)=f \mathrm{v}(z)
$$
$\Lambda=\int F(z) d z$ are related according to Eq. 9 by

The axially integrated concentration $A=\int c(z) d z$ and the axially integrated fluorescence

with the constant $\eta=\lambda \int \operatorname{RIPSF}(z) d z$.

\section{Z-scan and FFS acquisition in live cells}

484 Cells were selected by verifying the desired expression levels for the mCherry and EGFP labeled constructs using 1-photon epi illumination provided by a mercury lamp and using an EGFP/mCherry filter set. Points within cells for z-scan and FFS data collection were chosen where the fluorescence appeared locally ( $3 \mu \mathrm{m}$ 
radius) uniform as visualized in epi illumination and where a cell lacked nearby internal structures when visualized by using transmitted light. For data acquisition, the microscope was switched to two-photon mode and the excitation volume was focused to the middle of the cell. In most instances, $60 \mathrm{~s}$ of $z$-scan data or where increased statistical precision was desired.

\section{Effective diffusion coefficient}

The effective diffusion coefficient was calculated as (89) $D_{\text {eff }}=\omega_{0}^{2} /\left(8 \tau_{D}\right)$, where $\omega_{0}^{2}$ is the $1 / e^{2}$ radial

beam waist of the 2-photon excitation light focus and $\tau_{D}$ was estimated by fitting the log-averaged

autocorrelation of photon counts $G(\tau)$ to $G_{2 D}(\tau)=G_{0} /\left(1+\left(\tau / \tau_{D}\right)\right)^{-1}+G_{\text {offset }}$. FCS data quality control was performed by redacting data where $G_{\text {offset }}$ showed significantly negative values or where $\tau_{D}$ indicated by the fit was $>50 \%$ higher than the time at which the correlation amplitude reached half its initial value.

\section{Modeling of DC z-scan intensity profiles}

The DC fluorescence intensity $\mathbf{F}^{\mathbf{G}}(z)$ of a z-scan intensity profile of EGFP (G)-labeled protein through a series of horizontal layers, each containing a uniform concentration of labels, is described by (20-22)

$$
\mathbf{F}^{\mathbf{G}}(z)=\sum_{i} \mathbf{f}_{i}^{\mathrm{G}} \cdot \mathrm{v}\left(z ; a_{i}^{\mathrm{G}}, b_{i}^{\mathrm{G}}\right)=\boldsymbol{\alpha}^{\mathrm{G}} \sum_{i} f_{i}^{\mathrm{G}} \cdot \mathrm{v}\left(z ; a_{i}^{\mathrm{G}}, b_{i}^{\mathrm{G}}\right)
$$

where $f_{i}^{\mathrm{G}}$ and $\mathrm{v}\left(z, a_{i}^{\mathrm{G}}, b_{i}^{\mathrm{G}}\right)$ are the fluorescence amplitude and shape function associated with the $i$-th

layer. The shape function $\mathrm{v}\left(z, a_{i}^{\mathrm{G}}, b_{i}^{\mathrm{G}}\right)$ characterizes a uniform slab or $\mathrm{S}$ layer starting at $z=a_{i}^{\mathrm{G}}$ and ending at $z=b_{i}^{\mathrm{G}}$, while $\mathrm{v}\left(z, a_{i}^{\mathrm{G}}, a_{i}^{\mathrm{G}}\right)$ defines an infinitesimally thin or $\delta$ layer located at $z=a_{i}^{\mathrm{G}}(20-22)$.

Vector notation is used to describe properties of the green ( $g$ ) and red ( $r$ ) channel, i.e. $\mathbf{F}^{\mathbf{G}}(z)=\left(F_{g}^{\mathrm{G}}(z), F_{r}^{\mathrm{G}}(z)\right)$ and $\mathbf{f}_{i}^{\mathbf{G}}=\left(f_{i, g}^{\mathrm{G}}, f_{i, r}^{\mathrm{G}}\right)$. The broad emission spectrum of EGFP is split at the dichroic mirror, resulting in identical shapes of the z-scan profile in both channels. Their intensity ratio is specified by the crosstalk vector $\boldsymbol{\alpha}^{\mathbf{G}}$, which is defined by $\mathbf{f}_{i}^{\mathbf{G}}=\boldsymbol{\alpha}^{\mathbf{G}} f_{i}^{\mathrm{G}}$, where $f_{i}^{\mathrm{G}}=f_{i, g}^{\mathrm{G}}+f_{i, r}^{\mathrm{G}}$ is the sum 
amplitude of both channels. Similarly, the DC z-scan intensity profiles of the red fluorescent protein mCherry $(\mathrm{CH})$ are given by

$$
\mathbf{F}^{\mathrm{CH}}(z)=\sum_{i} \mathbf{f}_{i}^{\mathrm{CH}} \cdot \mathrm{v}\left(z ; a_{i}^{\mathrm{CH}}, b_{i}^{\mathrm{CH}}\right)=\boldsymbol{\alpha}^{\mathrm{CH}} \sum_{i} f_{i}^{\mathrm{CH}} \cdot \mathrm{v}\left(z ; a_{i}^{\mathrm{CH}}, b_{i}^{\mathrm{CH}}\right),
$$

and the presence of both proteins is described by the sum of Eqs. 12 and 13,

$$
\mathbf{F}(z)=\mathbf{F}^{\mathbf{G}}(z)+\mathbf{F}^{\mathbf{C H}}(z) .
$$

Only a few scan geometries are of interest for modeling of the data in this study. The first uses a single slab layer to describe the distribution of $\operatorname{EGFP}(X=G)$ and mCherry $(X=C H)$ labeled species,

$$
\mathbf{F}_{\mathbf{S}}^{\mathbf{x}}(z)=\boldsymbol{\alpha}^{\mathbf{x}} f^{\mathrm{x}} \mathbf{v}\left(z ; a^{\mathrm{x}}, b^{\mathrm{x}}\right)
$$

as illustrated in Fig. 1A. The second geometry consists of a delta layer followed by a slab layer and another

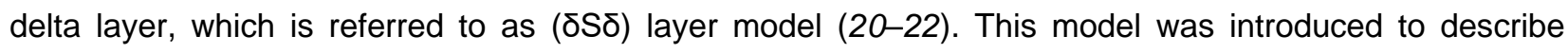
peripheral membrane proteins with the $\delta$ layers representing the top and bottom plasma membrane, while the S layer describes the cytoplasmic space (21). The DC z-scan ( $\delta S \delta)$ model is given by

$$
\mathbf{F}_{\delta \mathbf{s} \delta}^{\mathbf{x}}(z)=\boldsymbol{\alpha}^{\mathrm{x}}\left(f_{\mathrm{B}}^{\mathrm{x}} \cdot \mathrm{v}\left(z ; a^{\mathrm{x}}, a^{\mathrm{x}}\right)+f_{\mathrm{Cy}}^{\mathrm{x}} \cdot \mathrm{v}\left(z ; a^{\mathrm{x}}, b^{\mathrm{x}}\right)+f_{\mathrm{T}}^{\mathrm{x}} \cdot \mathrm{v}\left(z ; b^{\mathrm{x}}, b^{\mathrm{x}}\right)\right),
$$

where $a^{\mathrm{X}}$ and $b^{\mathrm{X}}$ mark the axial height of the bottom (B) and top (T) membrane (see Fig. 1G).

The fluorescence amplitudes of the $\mathrm{B}, \mathrm{T}$ and cytoplasmic (Cy) layer are given by $f_{\mathrm{B}}^{\mathrm{X}}, f_{\mathrm{T}}^{\mathrm{X}}$, and $f_{\mathrm{Cy}}^{\mathrm{X}}$.

The DC z-scan of cells co-expressing EGFP and mCherry labeled proteins is described by a superposition of their individual profiles (Eq. 14). The notation $X^{G}-Y^{C H}$ was used to describe a model where the z-scan intensity of the EGFP-labeled protein is described by the X profile, while the z-scan intensity of the mCherrylabeled protein is given by the $\mathrm{Y}$ profile,

$$
\mathbf{F}_{\mathbf{X}-\mathbf{Y}}(z)=\mathbf{F}_{\mathbf{X}}^{\mathbf{G}}(z)+\mathbf{F}_{\mathbf{Y}}^{\mathbf{C H}}(z)
$$

where $\mathrm{X}$ and $\mathrm{Y}$ denote either the $\mathrm{S}$ or $\delta S \delta$ model (Eqs. 15 and 16). For example, the $\mathrm{S}_{-}^{\mathrm{G}} \mathrm{S}^{\mathrm{CH}}$ model is based on independent slab layers for the EGFP and mCherry-labeled proteins, while the $\delta S \delta^{G}-S^{C H}$ model uses the ( $\delta S \delta)$ model for the EGFP label and a slab layer for mCherry. 


\section{Determining cell thickness from the z-scan intensity profile}

538 For a cell section that is thick with respect to the RIPSF width, a direct fit of the intensity profile to a slab 539 model (Eq. 15) determines the cell thickness by $L_{c e l l}=b^{C H}-a^{C H}$. For cell sections that are thin with respect 540 to the RIPSF, cell thickness cannot be reliably determined from fitting to a slab model (32). However, 541 because the fluorescent protein mCherry is distributed uniformly throughout the cell interior, its $z$-scan 542 intensity provides a measure of local cell thickness $L_{\text {cell. }}$ In this manner the cell thickness in thin sections can 543 be determined from the integrated fluorescence $\Lambda^{C H}$ of the intensity profile. $\Lambda^{C H}$ is proportional to the 544 integrated concentration area $A^{C H}$ (Eq. 11), which is $L_{c e l l} c^{C H}$ for the slab geometry, where the mCherry 545 concentration $c^{C H}$ is assumed constant throughout the cell. Thus, the ratio of the integrated intensity profiles 546 from a thick and a thin section measured in the same cell equals their thickness ratio,

$547 \quad \Lambda_{\text {thin }}^{C H} / \Lambda_{\text {thick }}^{C H}=L_{\text {cell,thin }} / L_{\text {cell,thick }}$, which was used to determine the thickness of thin cell sections. Although previous work (32) defined the cutoff between thick and thin cell lections as $L_{\text {cell }}=0.5 \mu \mathrm{m}$, a more conservative value of $1.5 \mu \mathrm{m}$ was used in this study to improve the precision of length measurements .

\section{Production of PM blebs}

Bulges along the plasma membrane (i.e., blebs) were induced along the PM of cultured HeLa cells by adding $10-15 \%$ ethanol (23) to the cell growth medium and incubating for $\sim 15 \mathrm{~min}$ at $37^{\circ} \mathrm{C}$ and $5 \% \mathrm{CO}_{2}$. The ethanoltreated cells were subsequently analyzed by transmitted light microscopy to assess the number and size of blebs with the goal of producing blebs with $\sim 5 \mu \mathrm{m}$ diameter in sufficient quantity for measurement. If insufficient blebbing was observed, cells were returned to the incubator for $\sim 10$ min before re-checking. Once sufficient blebbing was obtained, the growth medium with ethanol was exchanged by PBS containing ethanol immediately before measurement.

\section{SYTO RNASelect cell stain}

Live cells were stained with SYTO RNASelect (Thermo Fisher Scientific, Waltham, MA) by diluting the stock dye $1000 \mathrm{x}$ ( $5 \mu \mathrm{M}$ final concentration) into the cell culture medium followed by incubation for $1 \mathrm{~h}$ at $37^{\circ} \mathrm{C}$ and $5 \% \mathrm{CO}_{2}$. For measurement, the growth medium with SYTO RNASelect was replaced by PBS containing the same concentration of the dye. 


\section{Z-scan redaction and statistical analysis}

567 Despite the selection of scan locations as described earlier, some of the measured z-scan intensity profiles 568 were not adequately described by our fit model. This was attributed to scans that inadvertently passed 569 through complex intracellular structures. Since these structures are not captured by the model framework, the spatial autocorrelation of the normalized residual from the fit was calculated to identify patterns that are 571 indicative of the presence of extraneous cellular features not described by the fit model. Scans with a 572 correlation amplitude exceeding a threshold value were removed. Furthermore, z-scan measurements of 573 the cortex thickness were selected to have a cell thickness $L_{c e l l} \geq 2.5 \mu \mathrm{m}$ and a membrane fraction (22) $m \geq$ $574 \quad 0.5$ in order to ensure the absence of biases in the fitted values as supported by our study using simulated DC z-scans (Fig. S1). Quality control for the z-scan fits of cortex thickness was provided by averaging the normalized residuals within $\pm 1 \mu \mathrm{m}$ of the fitted PM locations. Scans where these averaged residuals individually, yielding 12 estimates of each parameter of interest. This set of parameters served to calculate

580 the average effective depletion length $\bar{\Delta}_{i}$ and its standard deviation $\sigma_{i}$ at the $i$-th z-scan location. Multiple

measurement locations were summarized using the weighted average $\langle\Delta\rangle$ over all locations with $1 / \sigma_{i}^{2}$ as weight. The uncertainty in the weighted average was computed using the dispersion corrected standard error of the mean (SEM),

where $\sigma_{w e}^{2}$ is the variance of the weighted average. Since it was observed that $\langle\Delta\rangle$ at the top $(\mathrm{T})$ and bottom

(B) PM interfaces are in agreement $\left(\left\langle\Delta^{T}\right\rangle \approx\left\langle\Delta^{B}\right\rangle\right)$, data from both interfaces were grouped together when 587 calculating $\langle\Delta\rangle$. This is indicated by dropping the $T$ and $B$ superscript.

\section{Simulations of z-scan traces}


Simulated DC z-scan traces were obtained following a previously described procedure $(20)$ to evaluate models and estimate the statistical uncertainty in DC z-scan fit results. Simulated DC z-scan traces were fit using the same algorithms that were applied to experimental data. All data derived from simulations represent the average result from fits of $n=1000 \mathrm{z}$-scan traces.

\section{References}

596 1. P. Chugh, E. K. Paluch, The actin cortex at a glance. J Cell Sci. 131 (2018),

597 doi:10.1242/jcs.186254.

598 2. A. Kusumi, T. K. Fujiwara, R. Chadda, M. Xie, T. A. Tsunoyama, Z. Kalay, R. S. Kasai,

599 K. G. N. Suzuki, Dynamic organizing principles of the plasma membrane that regulate signal

600 transduction: commemorating the fortieth anniversary of Singer and Nicolson's fluid-mosaic

601 model. Annu Rev Cell Dev Biol. 28, 215-250 (2012).

602 3. M. D. Blower, Molecular insights into intracellular RNA localization. Int Rev Cell Mol

603 Biol. 302, 1-39 (2013).

604 4. C. Medioni, K. Mowry, F. Besse, Principles and roles of mRNA localization in animal 605 development. Development. 139, 3263-3276 (2012).

606 5. E. Lécuyer, H. Yoshida, N. Parthasarathy, C. Alm, T. Babak, T. Cerovina, T. R. Hughes, 607 P. Tomancak, H. M. Krause, Global analysis of mRNA localization reveals a prominent role in 608 organizing cellular architecture and function. Cell. 131, 174-187 (2007).

609 6. A. Gopal, Z. H. Zhou, C. M. Knobler, W. M. Gelbart, Visualizing large RNA molecules in 610 solution. RNA. 18, 284-299 (2012).

611 7. A. Borodavka, S. W. Singaram, P. G. Stockley, W. M. Gelbart, A. Ben-Shaul, R. Tuma, 612 Sizes of Long RNA Molecules Are Determined by the Branching Patterns of Their Secondary 613 Structures. Biophys J. 111, 2077-2085 (2016).

614 8. S. S. Sommer, J. E. Cohen, The size distributions of proteins, mRNA, and nuclear RNA. J 615 Mol Evol. 15, 37-57 (1980). 
$6169 . \quad$ I. Legnini, J. Alles, N. Karaiskos, S. Ayoub, N. Rajewsky, FLAM-seq: full-length mRNA 617 sequencing reveals principles of poly(A) tail length control. Nat Methods. 16, 879-886 (2019).

618 10. M. F. Tam, J. A. Dodd, W. E. Hill, Physical characteristics of 16 S rRNA under 619 reconstitution conditions. J Biol Chem. 256, 6430-6434 (1981).

620 11. A. Verschoor, J. Frank, Three-dimensional structure of the mammalian cytoplasmic 621 ribosome. J Mol Biol. 214, 737-749 (1990).

622 12. G. T. Charras, T. J. Mitchison, L. Mahadevan, Animal cell hydraulics. J Cell Sci. 122, $623 \quad 3233-3241(2009)$

624 13. E. Moeendarbary, L. Valon, M. Fritzsche, A. R. Harris, D. A. Moulding, A. J. Thrasher, E. 625 Stride, L. Mahadevan, G. T. Charras, The cytoplasm of living cells behaves as a poroelastic 626 material. Nat Mater. 12, 253-261 (2013).

627 14. K. Luby-Phelps, P. E. Castle, D. L. Taylor, F. Lanni, Hindered diffusion of inert tracer 628 particles in the cytoplasm of mouse 3T3 cells. Proc Natl Acad Sci U S A. 84, 4910-4913 (1987).

629 15. K. Luby-Phelps, D. L. Taylor, Subcellular compartmentalization by local differentiation of 630 cytoplasmic structure. Cell Motil Cytoskeleton. 10, 28-37 (1988).

631 16. L. Hou, F. Lanni, K. Luby-Phelps, Tracer diffusion in F-actin and Ficoll mixtures. Toward 632 a model for cytoplasm. Biophys J. 58, 31-43 (1990).

633 17. G. L. Lukacs, P. Haggie, O. Seksek, D. Lechardeur, N. Freedman, A. S. Verkman, Size634 dependent DNA mobility in cytoplasm and nucleus. J Biol Chem. 275, 1625-1629 (2000).

635 18. E. Dauty, A. S. Verkman, Actin cytoskeleton as the principal determinant of size636 dependent DNA mobility in cytoplasm: a new barrier for non-viral gene delivery. J Biol Chem. $637 \quad 280,7823-7828(2005)$.

638 19. K. Luby-Phelps, Effect of cytoarchitecture on the transport and localization of protein 639 synthetic machinery. Journal of Cellular Biochemistry. 52, 140-147 (1993). 
640 20. I. Angert, S. R. Karuka, J. Hennen, Y. Chen, J. P. Albanesi, L. M. Mansky, J. D. Mueller, 641 Sensitive Detection of Protein Binding to the Plasma Membrane with Dual-Color Z-Scan

642 Fluorescence. Biophys J. 118, 281-293 (2020).

643 21. E. M. Smith, P. J. Macdonald, Y. Chen, J. D. Mueller, Quantifying protein-protein 644 interactions of peripheral membrane proteins by fluorescence brightness analysis. Biophys J. 107, $645 \quad 66-75(2014)$

646 22. E. M. Smith, J. Hennen, Y. Chen, J. D. Mueller, Z-scan fluorescence profile

647 deconvolution of cytosolic and membrane-associated protein populations. Anal Biochem. 480, $648 \quad 11-20(2015)$

649 23. S. Saha, I.-H. Lee, A. Polley, J. T. Groves, M. Rao, S. Mayor, Diffusion of GPI-anchored 650 proteins is influenced by the activity of dynamic cortical actin. Mol Biol Cell. 26, 4033-4045 651 (2015).

652 24. R. E. Scott, R. G. Perkins, M. A. Zschunke, B. J. Hoerl, P. B. Maercklein, Plasma 653 membrane vesiculation in 3T3 and SV3T3 cells. I. Morphological and biochemical 654 characterization. J Cell Sci. 35, 229-243 (1979).

655 25. T. Baumgart, A. T. Hammond, P. Sengupta, S. T. Hess, D. A. Holowka, B. A. Baird, W. 656 W. Webb, Large-scale fluid/fluid phase separation of proteins and lipids in giant plasma 657 membrane vesicles. Proc Natl Acad Sci U S A. 104, 3165-3170 (2007).

658 26. F. Schneider, D. Waithe, M. P. Clausen, S. Galiani, T. Koller, G. Ozhan, C. Eggeling, E. 659 Sezgin, Diffusion of lipids and GPI-anchored proteins in actin-free plasma membrane vesicles 660 measured by STED-FCS. Mol Biol Cell. 28, 1507-1518 (2017).

661 27. T. M. Svitkina, Actin Cell Cortex: Structure and Molecular Organization. Trends Cell 662 Biol. 30, 556-565 (2020). 
663 28. N. Morone, T. Fujiwara, K. Murase, R. S. Kasai, H. Ike, S. Yuasa, J. Usukura, A. Kusumi, 664 Three-dimensional reconstruction of the membrane skeleton at the plasma membrane interface by 665 electron tomography. J Cell Biol. 174, 851-862 (2006).

666 29. P. Chugh, A. G. Clark, M. B. Smith, D. A. D. Cassani, K. Dierkes, A. Ragab, P. P. Roux, 667 G. Charras, G. Salbreux, E. K. Paluch, Actin cortex architecture regulates cell surface tension. $668 \quad$ Nat Cell Biol. 19, 689-697 (2017).

669 30. A. G. Clark, K. Dierkes, E. K. Paluch, Monitoring actin cortex thickness in live cells. 670 Biophys J. 105, 570-580 (2013).

671 31. J. Riedl, A. H. Crevenna, K. Kessenbrock, J. H. Yu, D. Neukirchen, M. Bista, F. Bradke, 672 D. Jenne, T. A. Holak, Z. Werb, M. Sixt, R. Wedlich-Soldner, Lifeact: a versatile marker to 673 visualize F-actin. Nat Methods. 5, 605-607 (2008).

674 32. P. J. Macdonald, Y. Chen, X. Wang, Y. Chen, J. D. Mueller, Brightness analysis by Z675 scan fluorescence fluctuation spectroscopy for the study of protein interactions within living cells. 676 Biophys J. 99, 979-988 (2010).

677 33. P. R. Tedbury, E. O. Freed, HIV-1 gag: an emerging target for antiretroviral therapy. Curr Top Microbiol Immunol. 389, 171-201 (2015).

679 34. J. G. Levin, J. Guo, I. Rouzina, K. Musier-Forsyth, Nucleic acid chaperone activity of 680 HIV-1 nucleocapsid protein: critical role in reverse transcription and molecular mechanism. Prog $681 \quad$ Nucleic Acid Res Mol Biol. 80, 217-286 (2005).

682 35. R. N. De Guzman, Z. R. Wu, C. C. Stalling, L. Pappalardo, P. N. Borer, M. F. Summers, 683 Structure of the HIV-1 nucleocapsid protein bound to the SL3 psi-RNA recognition element. 684 Science. 279, 384-388 (1998).

685 36. A. Rein, Nucleic acid chaperone activity of retroviral Gag proteins. RNA Biol. 7, 700-705 686 (2010). 
687 37. V. Chukkapalli, S. J. Oh, A. Ono, Opposing mechanisms involving RNA and lipids 688 regulate HIV-1 Gag membrane binding through the highly basic region of the matrix domain. 689 Proc Natl Acad Sci U S A. 107, 1600-1605 (2010).

690 38. P. Purohit, S. Dupont, M. Stevenson, M. R. Green, Sequence-specific interaction between 691 HIV-1 matrix protein and viral genomic RNA revealed by in vitro genetic selection. RNA. 7, 576$692584(2001)$

693 39. D. Thornhill, B. Olety, A. Ono, Relationships between MA-RNA Binding in Cells and 694 Suppression of HIV-1 Gag Mislocalization to Intracellular Membranes. J Virol. 93 (2019), 695 doi:10.1128/JVI.00756-19.

696 40. C. R. Gaines, E. Tkacik, A. Rivera-Oven, P. Somani, A. Achimovich, T. Alabi, A. Zhu, N. 697 Getachew, A. L. Yang, M. McDonough, T. Hawkins, Z. Spadaro, M. F. Summers, HIV-1 Matrix 698 Protein Interactions with tRNA: Implications for Membrane Targeting. J Mol Biol. 430, 2113$6992127(2018)$

700 41. S. B. Kutluay, T. Zang, D. Blanco-Melo, C. Powell, D. Jannain, M. Errando, P. D.

701 Bieniasz, Global changes in the RNA binding specificity of HIV-1 gag regulate virion genesis.

702 Cell. 159, 1096-1109 (2014).

703 42. Y. Iwatani, H. Takeuchi, K. Strebel, J. G. Levin, Biochemical activities of highly purified, 704 catalytically active human APOBEC3G: correlation with antiviral effect. J Virol. 80, 5992-6002 705 (2006).

706 43. Y. N. Friew, V. Boyko, W.-S. Hu, V. K. Pathak, Intracellular interactions between APOBEC3G, RNA, and HIV-1 Gag: APOBEC3G multimerization is dependent on its association with RNA. Retrovirology. 6, 56 (2009).

44. J. Li, Y. Chen, M. Li, M. A. Carpenter, R. M. McDougle, E. M. Luengas, P. J. Macdonald, R. S. Harris, J. D. Mueller, APOBEC3 multimerization correlates with HIV-1 packaging and 711 restriction activity in living cells. J Mol Biol. 426, 1296-1307 (2014). 
712 45. E. S. Svarovskaia, H. Xu, J. L. Mbisa, R. Barr, R. J. Gorelick, A. Ono, E. O. Freed, W.-S.

$713 \mathrm{Hu}, \mathrm{V} . \mathrm{K}$. Pathak, Human apolipoprotein B mRNA-editing enzyme-catalytic polypeptide-like 3G

714 (APOBEC3G) is incorporated into HIV-1 virions through interactions with viral and nonviral

715 RNAs. J Biol Chem. 279, 35822-35828 (2004).

716 46. A. Burnett, P. Spearman, APOBEC3G multimers are recruited to the plasma membrane

717 for packaging into human immunodeficiency virus type 1 virus-like particles in an RNA-

718 dependent process requiring the NC basic linker. J Virol. 81, 5000-5013 (2007).

719 47. B. R. Cullen, Role and mechanism of action of the APOBEC3 family of antiretroviral

720 resistance factors. J Virol. 80, 1067-1076 (2006).

721 48. J. S. Albin, R. S. Harris, Interactions of host APOBEC3 restriction factors with HIV-1 in 722 vivo: implications for therapeutics. Expert Rev Mol Med. 12, e4 (2010).

723 49. J. F. Hultquist, J. A. Lengyel, E. W. Refsland, R. S. LaRue, L. Lackey, W. L. Brown, R. S.

724 Harris, Human and rhesus APOBEC3D, APOBEC3F, APOBEC3G, and APOBEC3H

725 demonstrate a conserved capacity to restrict Vif-deficient HIV-1. J Virol. 85, 11220-11234

726 (2011).

727 50. L. W. Janson, K. Ragsdale, K. Luby-Phelps, Mechanism and size cutoff for steric

728 exclusion from actin-rich cytoplasmic domains. Biophys J. 71, 1228-1234 (1996).

729 51. A. P. Minton, Confinement as a determinant of macromolecular structure and reactivity.

730 Biophys J. 63, 1090-1100 (1992).

731 52. J. C. Giddings, E. Kucera, C. P. Russell, M. N. Myers, Statistical theory for the

732 equilibrium distribution of rigid molecules in inert porous networks. Exclusion chromatography.

733 J. Phys. Chem. 72, 4397-4408 (1968).

734 53. T. Kalwarczyk, N. Ziebacz, A. Bielejewska, E. Zaboklicka, K. Koynov, J. Szymański, A.

735 Wilk, A. Patkowski, J. Gapiński, H.-J. Butt, R. Hołyst, Comparative analysis of viscosity of 
736 complex liquids and cytoplasm of mammalian cells at the nanoscale. Nano Lett. 11, 2157-2163

737 (2011).

738 54. R. Holyst, A. Bielejewska, J. Szymański, A. Wilk, A. Patkowski, J. Gapiński, A.

739 Zywociński, T. Kalwarczyk, E. Kalwarczyk, M. Tabaka, N. Ziebacz, S. A. Wieczorek, Scaling

740 form of viscosity at all length-scales in poly(ethylene glycol) solutions studied by fluorescence

741 correlation spectroscopy and capillary electrophoresis. Phys Chem Chem Phys. 11, 9025-9032

742 (2009).

743 55. L. Xiang, K. Chen, R. Yan, W. Li, K. Xu, Single-molecule displacement mapping unveils

744 nanoscale heterogeneities in intracellular diffusivity. Nat Methods. 17, 524-530 (2020).

745 56. T. P. Stossel, P. A. Janmey, K. S. Zaner, in Cytomechanics: The Mechanical Basis of Cell

746 Form and Structure, J. Bereiter-Hahn, O. R. Anderson, W.-E. Reif, Eds. (Springer, Berlin,

747 Heidelberg, 1987; https://doi.org/10.1007/978-3-642-72863-1_9), pp. 131-153.

748 57. J.-F. Joanny, J. Prost, Active gels as a description of the actin-myosin cytoskeleton. HFSP

$749 \quad J .3,94-104(2009)$.

750 58. T. C. Laurent, J. Killander, A theory of gel filtration and its exeperimental verification.

751 Journal of Chromatography A. 14, 317-330 (1964).

$752 \quad 59 . \quad$ K. C. Duong-Ly, S. B. Gabelli, Gel filtration chromatography (size exclusion

753 chromatography) of proteins. Methods Enzymol. 541, 105-114 (2014).

754 60. T. Kaur, I. Alshareedah, W. Wang, J. Ngo, M. M. Moosa, P. R. Banerjee, Molecular

755 Crowding Tunes Material States of Ribonucleoprotein Condensates. Biomolecules. 9 (2019),

756 doi:10.3390/biom9020071.

757 61. A. A. M. André, E. Spruijt, Liquid-Liquid Phase Separation in Crowded Environments. Int

$758 \quad J$ Mol Sci. 21 (2020), doi:10.3390/ijms21165908.

759 62. D. W. Provance, A. McDowall, M. Marko, K. Luby-Phelps, Cytoarchitecture of size-

760 excluding compartments in living cells. J Cell Sci. 106 ( Pt 2), 565-577 (1993). 
761 63. K. Luby-Phelps, D. L. Taylor, F. Lanni, Probing the structure of cytoplasm. J Cell Biol. $762 \mathbf{1 0 2}, 2015-2022(1986)$.

763 64. R. Kumar, S. Saha, B. Sinha, Cell spread area and traction forces determine myosin-II764 based cortex thickness regulation. Biochim Biophys Acta Mol Cell Res. 1866, 118516 (2019).

765 65. T. M. Svitkina, A. A. Shevelev, A. D. Bershadsky, V. I. Gelfand, Cytoskeleton of mouse 766 embryo fibroblasts. Electron microscopy of platinum replicas. Eur J Cell Biol. 34, 64-74 (1984).

767 66. F. Feijó Delgado, N. Cermak, V. C. Hecht, S. Son, Y. Li, S. M. Knudsen, S. Olcum, J. M. 768 Higgins, J. Chen, W. H. Grover, S. R. Manalis, Intracellular water exchange for measuring the 769 dry mass, water mass and changes in chemical composition of living cells. PLoS One. 8, e67590 770 (2013).

771 67. M. A. Urbaneja, B. P. Kane, D. G. Johnson, R. J. Gorelick, L. E. Henderson, J. R. Casas772 Finet, Binding properties of the human immunodeficiency virus type 1 nucleocapsid protein $\mathrm{p} 7$ to 773 a model RNA: elucidation of the structural determinants for function. J Mol Biol. 287, 59-75 774 (1999).

775 68. W. Ouyang, S. Okaine, M. P. McPike, Y. Lin, P. N. Borer, Probing the RNA binding 776 surface of the HIV-1 nucleocapsid protein by site-directed mutagenesis. Biochemistry. 52, 3358$7773368(2013)$

778 69. M. Comas-Garcia, S. A. Datta, L. Baker, R. Varma, P. R. Gudla, A. Rein, Dissection of 779 specific binding of HIV-1 Gag to the "packaging signal" in viral RNA. Elife. 6 (2017), 780 doi:10.7554/eLife.27055.

781 70. M. Delarue, G. P. Brittingham, S. Pfeffer, I. V. Surovtsev, S. Pinglay, K. J. Kennedy, M. 782 Schaffer, J. I. Gutierrez, D. Sang, G. Poterewicz, J. K. Chung, J. M. Plitzko, J. T. Groves, C. 783 Jacobs-Wagner, B. D. Engel, L. J. Holt, mTORC1 Controls Phase Separation and the Biophysical 784 Properties of the Cytoplasm by Tuning Crowding. Cell. 174, 338-349.e20 (2018). 
785 71. K. Kwapiszewska, K. Szczepański, T. Kalwarczyk, B. Michalska, P. Patalas-Krawczyk, J. 786 Szymański, T. Andryszewski, M. Iwan, J. Duszyński, R. Hołyst, Nanoscale Viscosity of 787 Cytoplasm Is Conserved in Human Cell Lines. J Phys Chem Lett. 11, 6914-6920 (2020). 788 72. J. Chen, D. Grunwald, L. Sardo, A. Galli, S. Plisov, O. A. Nikolaitchik, D. Chen, S.

789 Lockett, D. R. Larson, V. K. Pathak, W.-S. Hu, Cytoplasmic HIV-1 RNA is mainly transported 790 by diffusion in the presence or absence of Gag protein. Proc Natl Acad Sci U S A. 111, E5205$7915213(2014)$

792 73. J. J. Blum, G. Lawler, M. Reed, I. Shin, Effect of cytoskeletal geometry on intracellular 793 diffusion. Biophys J. 56, 995-1005 (1989).

794 74. K. Luby-Phelps, F. Lanni, D. L. Taylor, The submicroscopic properties of cytoplasm as a 795 determinant of cellular function. Annu Rev Biophys Biophys Chem. 17, 369-396 (1988).

796 75. D. S. Banks, C. Fradin, Anomalous diffusion of proteins due to molecular crowding. 797 Biophys J. 89, 2960-2971 (2005).

798 76. A. Miermont, F. Waharte, S. Hu, M. N. McClean, S. Bottani, S. Léon, P. Hersen, Severe 799 osmotic compression triggers a slowdown of intracellular signaling, which can be explained by 800 molecular crowding. Proc Natl Acad Sci U S A. 110, 5725-5730 (2013).

801 77. M. Weiss, M. Elsner, F. Kartberg, T. Nilsson, Anomalous subdiffusion is a measure for 802 cytoplasmic crowding in living cells. Biophys J. 87, 3518-3524 (2004).

803 78. C. I. Lacayo, J. A. Theriot, Listeria monocytogenes actin-based motility varies depending 804 on subcellular location: a kinematic probe for cytoarchitecture. Mol Biol Cell. 15, 2164-2175 805 (2004).

$80679 . \quad$ J.-Y. Youn, B. J. A. Dyakov, J. Zhang, J. D. R. Knight, R. M. Vernon, J. D. Forman-Kay, 807 A.-C. Gingras, Properties of Stress Granule and P-Body Proteomes. Mol Cell. 76, 286-294 808 (2019). 
809 80. A. A. Hyman, C. A. Weber, F. Jülicher, Liquid-liquid phase separation in biology. Annu $810 \quad$ Rev Cell Dev Biol. 30, 39-58 (2014).

811 81. L.-P. Bergeron-Sandoval, N. Safaee, S. W. Michnick, Mechanisms and Consequences of 812 Macromolecular Phase Separation. Cell. 165, 1067-1079 (2016).

813 82. R. Wollman, T. Meyer, Coordinated oscillations in cortical actin and Ca2+ correlate with 814 cycles of vesicle secretion. Nat Cell Biol. 14, 1261-1269 (2012).

815 83. P. Li, A. T. Bademosi, J. Luo, F. A. Meunier, Actin Remodeling in Regulated Exocytosis: 816 Toward a Mesoscopic View. Trends Cell Biol. 28, 685-697 (2018).

817 84. A. Derdowski, L. Ding, P. Spearman, A novel fluorescence resonance energy transfer 818 assay demonstrates that the human immunodeficiency virus type 1 Pr55Gag I domain mediates 819 Gag-Gag interactions. J Virol. 78, 1230-1242 (2004).

820 85. C. Thaler, S. V. Koushik, H. L. Puhl, P. S. Blank, S. S. Vogel, Structural rearrangement of 821 CaMKIIalpha catalytic domains encodes activation. Proc Natl Acad Sci U S A. 106, 6369-6374 822 (2009).

823 86. B. Wu, Y. Chen, J. D. Müller, Fluorescence fluctuation spectroscopy of mCherry in living 824 cells. Biophys J. 96, 2391-2404 (2009).

825 87. B. Wu, Y. Chen, J. D. Müller, Heterospecies partition analysis reveals binding curve and 826 stoichiometry of protein interactions in living cells. Proc Natl Acad Sci U S A. 107, 4117-4122 827 (2010).

828 88. Y. Chen, H.-Q. Sun, J. P. Eichorst, J. P. Albanesi, H. Yin, J. D. Mueller, Comobility of 829 GABARAP and Phosphatidylinositol 4-Kinase 2A on Cytoplasmic Vesicles. Biochemistry. 57, $830 \quad 3556-3559(2018)$

831 89. J. D. Müller, Y. Chen, E. Gratton, Fluorescence correlation spectroscopy. Methods 832 Enzymol. 361, 69-92 (2003). 


\section{Acknowledgments}

836 Non-author contributions: The authors thank Yan Chen for help with logistics and experiment planning.

837 Author contributions: CIA, JDM, and SRK developed the dual color z-scan method. CIA and SRK 838 performed experiments and acquired the data. LMM helped interpret the data and guide the project. CIA 839 and JDM wrote analysis code, performed data analysis, and drafted the manuscript. All authors reviewed 840 and revised the manuscript.

841 Funding: This work was supported by NIH grants GM064589, GM124279 and GM098550. C. I. A. was 842 supported by NIH T32 AI083196

843 Competing interests: The authors declare that they have no financial or other competing interests.

844 Data and materials availability: The raw data and code necessary to perform the analyses will be made 845 available through the Dryad data repository. 
A mCherry-RXR \& EGFP

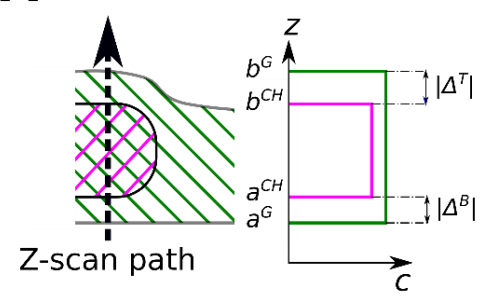

D

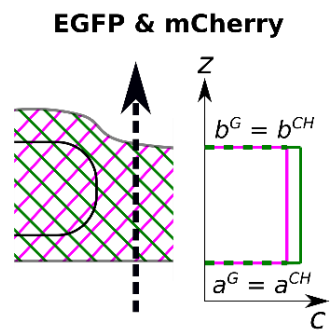

\section{G}

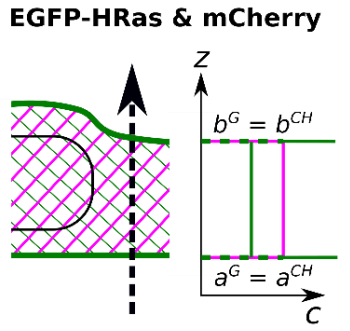

\section{J}

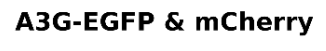
mCherry.

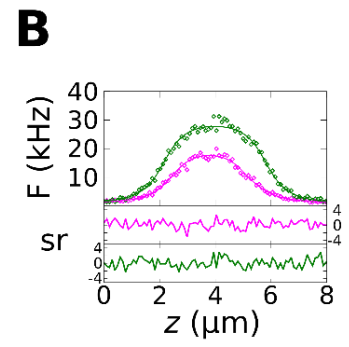

E
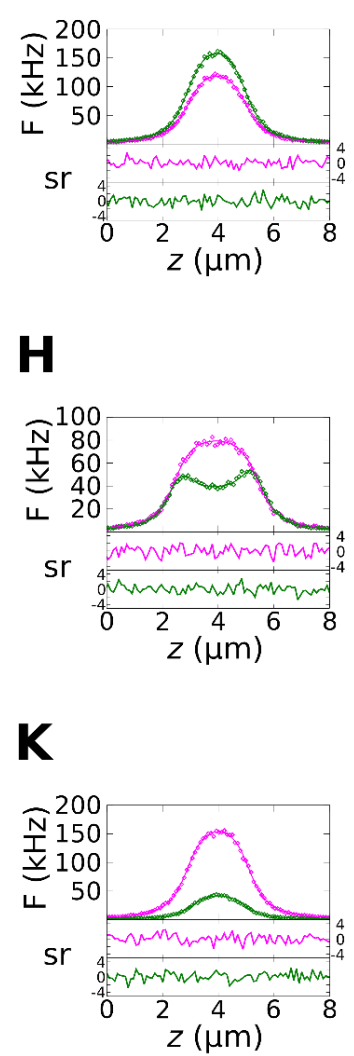

C

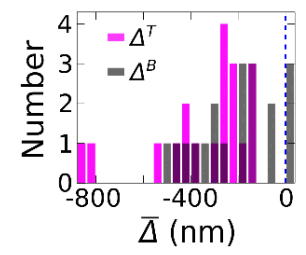

$\mathbf{F}$

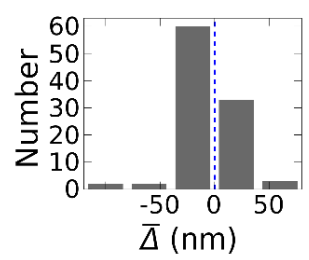

I

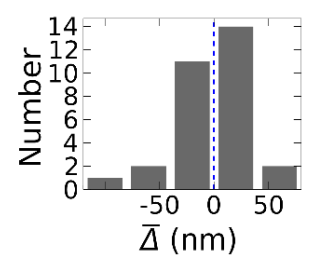

$\mathbf{L}$

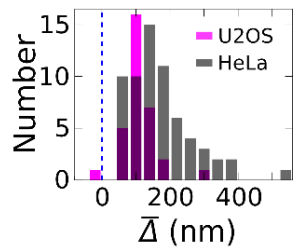

Fig. 1: DC z-scans in living cells. The z-scan trajectory through the cell is depicted in the cartoons along with crosshatched regions indicating the subcellular localization of the EGFP- and mCherry-labeled proteins (A, D, G, J, left panel) together with the concentration profile indicated by z-scan fitting for the EGFP (green lines) and mCherry (magenta lines) protein species (A, D, G, J, right panel). B, E, H, K) Fits (solid lines) of representative z-scan intensity traces of the green (green diamonds) and red (magenta diamonds) detection channel are shown along with their normalized residuals sr. The cell edge locations $a^{G}, b^{G}, a^{C H}, b^{C H}$ extracted from the fits determine the vertical distance $\Delta$ separating the EGFP- and mCherry-labeled slab edges in each z-scan. C, F, I, L) Histograms of $\bar{\Delta}$ from repeated z-scan experiments in many cells. Experiments with different EGFP- and mCherry-labeled protein species are indicated in rows: (A, B, C) EGFP and mCherryRXR, (D, E, F) EGFP and mCherry, (G, H, I) EGFP-HRas and mCherry, and (J, K, L) A3G-EGFP and 
A

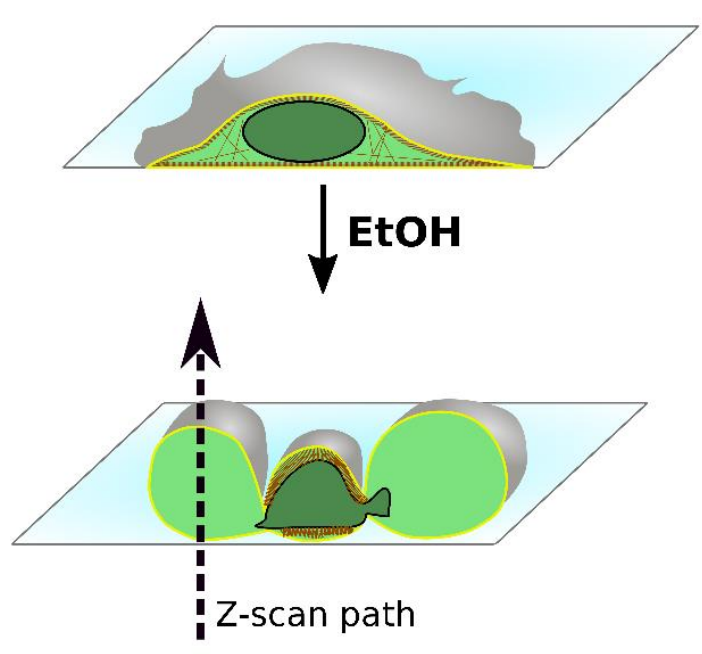

B

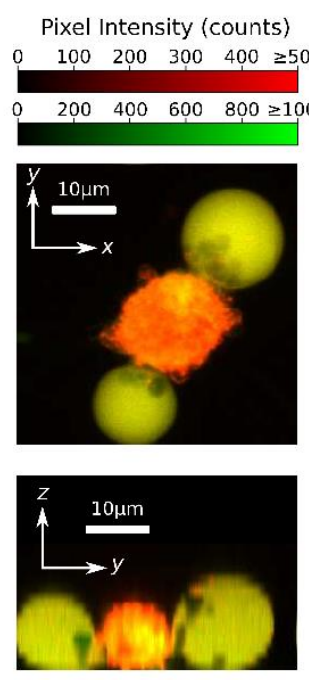

C

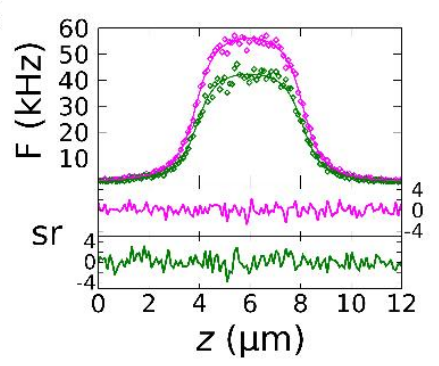

D

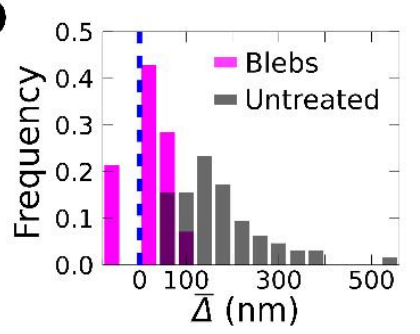

Fig. 2: Z-scans in blebs produced from HeLa cells co-expressing A3G-EGFP and mCherry. A) A

862 graphic depicting the production of large PM blebs from adherent cells via ethanol treatment is shown. In

863 this example, the cytoskeleton is denoted by brown colors. B) A DC 2-photon laser scanning z-stack image

864 of a HeLa cell with two large PM blebs is shown. The $x-y$ image was created by the summation of the z-

865 stack over the $z$-dimension. The $y-z$ image was produced by the sum of the $x$-dimension over two volume-

866 sections passing roughly through the center of each bleb. While significant red autofluorescence is produced

867 by the ethanol treatment near the cell center, it is typically absent in the blebs. Z-scan locations were selected

868 to pass near the center of each bleb, while avoiding regions possessing autofluorescence or identifiable

869 structures. C) Fit of a representative $z$-scan passing through a HeLa cell bleb $\left(X^{2}{ }^{2}=1.2\right)$. D) Histogram of $\bar{\Delta}$ values in the cytoplasm of HeLa cells not treated with ethanol (magenta bars) and in blebs produced by ethanol treatment (gray bars). 


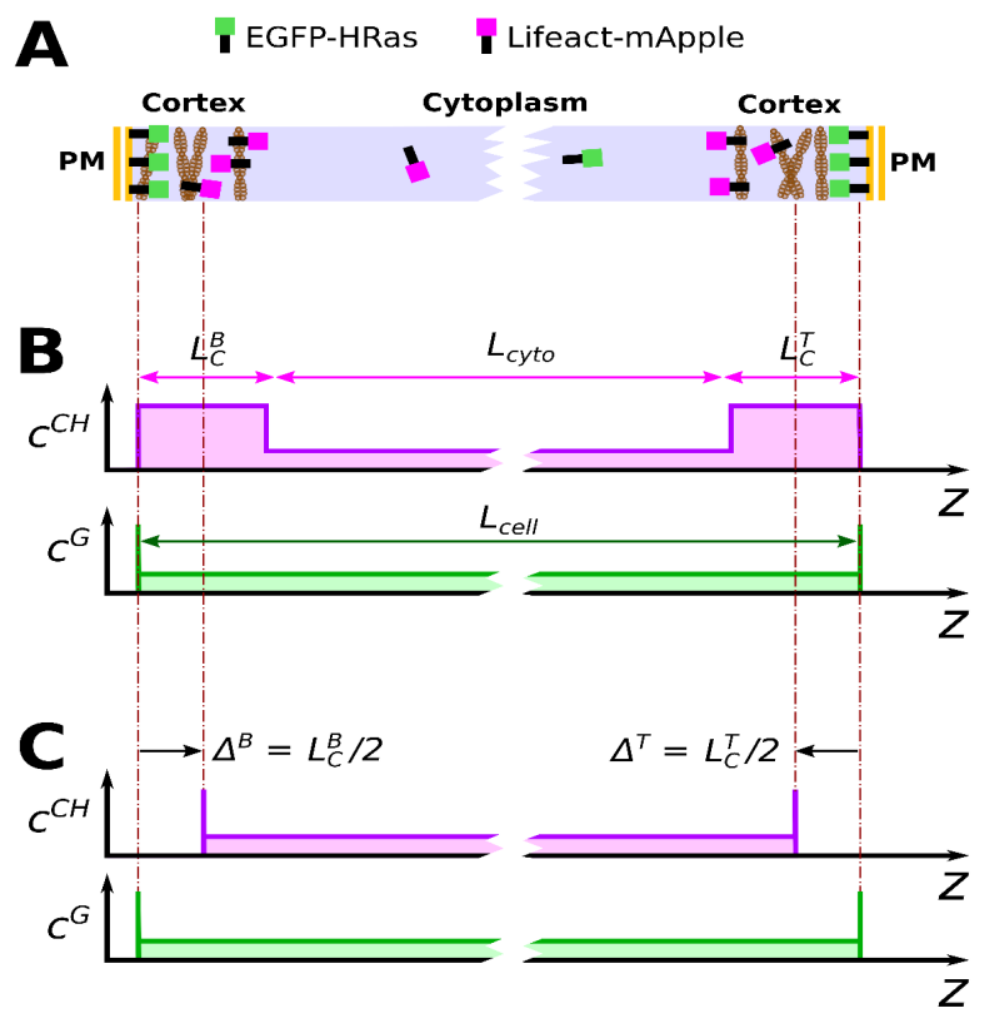
from the data in panel $(E)$.
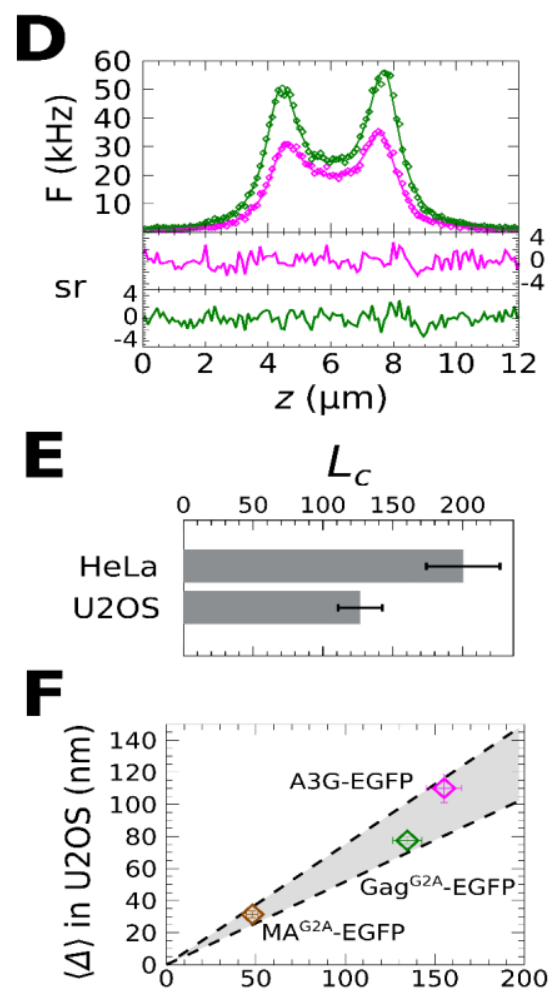

$\langle\Delta\rangle$ in HeLa $(\mathrm{nm})$

Fig. 3: DC z-scan measurement of cortex thickness. A) Lifeact-mApple demarcates the actin cortex, and EGFP-HRas demarcates the PM location. These fluorescent proteins lead to the $S S S^{C H}{ }_{-} \delta S \delta^{G}$ concentration profile depicted in (B). C) DC z-scans were fit to a $\delta S \delta^{C H}-\delta S \delta^{G}$ model. The location of the mApple $\delta$ layers corresponds to the centroid of the cortical slabs, so that the effective depletion length is half the thickness of the cortex slab. D) A fit of a DC z-scan through a HeLa cell co-expressing EGFP-HRas and LifeactmApple. E) Average cortex thickness values estimated by DC z-scan in HeLa and U2OS cells. F) $<\Delta>$ is

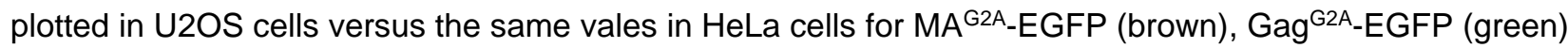
and A3G-EGFP (magenta). The ratio of cortex thicknesses in the cell lines corresponds to the slope of the data as is indicated by the shaded area and dashed lines, which correspond to the slope-uncertainty derived 

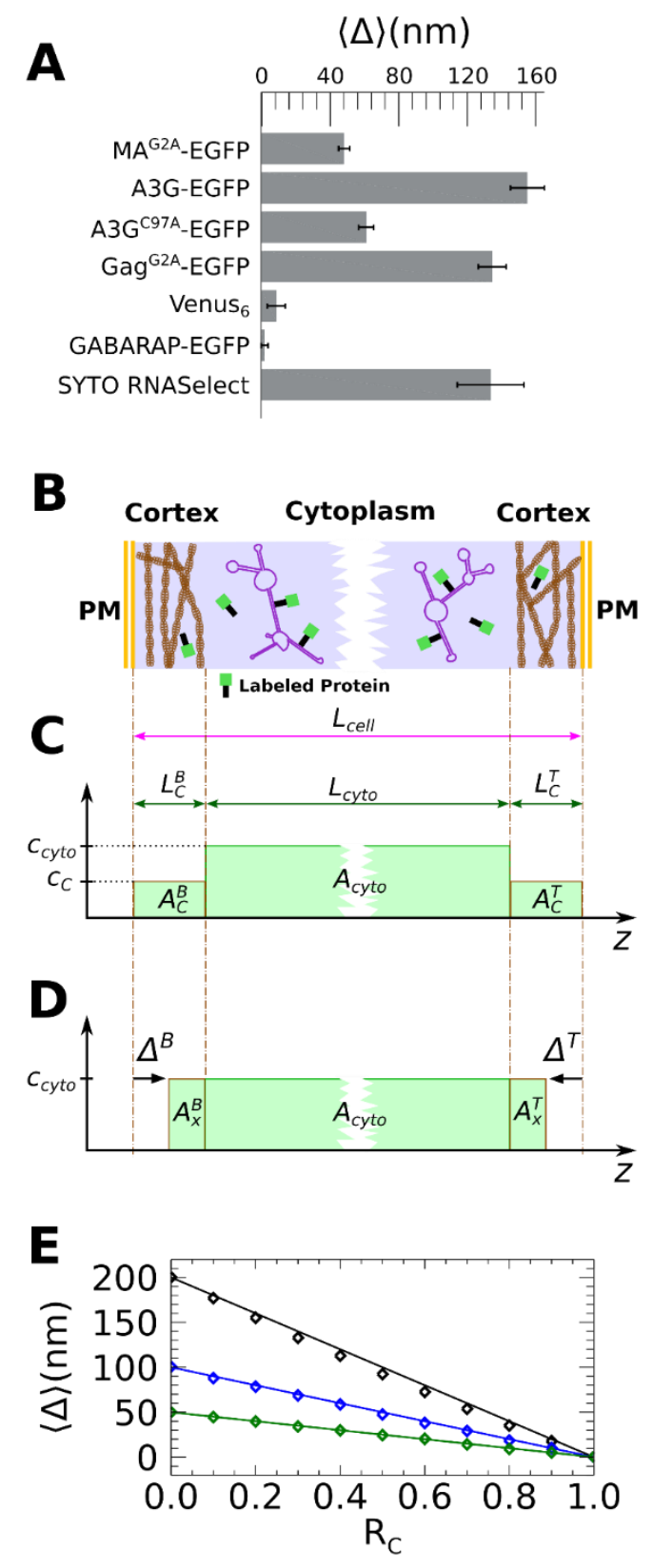

890 Fig. 4 Effective depletion length. A) $<\Delta>$ in HeLa cells for the indicated proteins and an RNA dye. B) Model 891 of cortical partitioning leading to a $S^{G} S^{G}$ distribution of fluorescence. C) Axial concentration profile 892 corresponding to the model. The concentration drops from $c_{c y t o}$ in the cytoplasm to $c_{c}$ in the cortex. D) 893 Approximation of the $S S S^{G}$ by a single $S^{G}$ concentration profile. Fitting of the experimental z-scan data to 894 this profile was used to identify apparent depletion lengths $\Delta^{\mathrm{B}}$ and $\Delta^{\mathrm{T}}$. The mCherry concentration profile 895 has been omitted in panels $C$ and D. E) $<\Delta>$ vs partition coefficient $R$ c extracted from simulated DC z-scans 896 for three different cortex lengths $\left(L_{C}^{B}=L_{C}^{T}\right.$ of 200,100 , and $\left.50 \mathrm{~nm}\right)$. The solid lines represent a theoretical 897 model based on Eq. 1. 

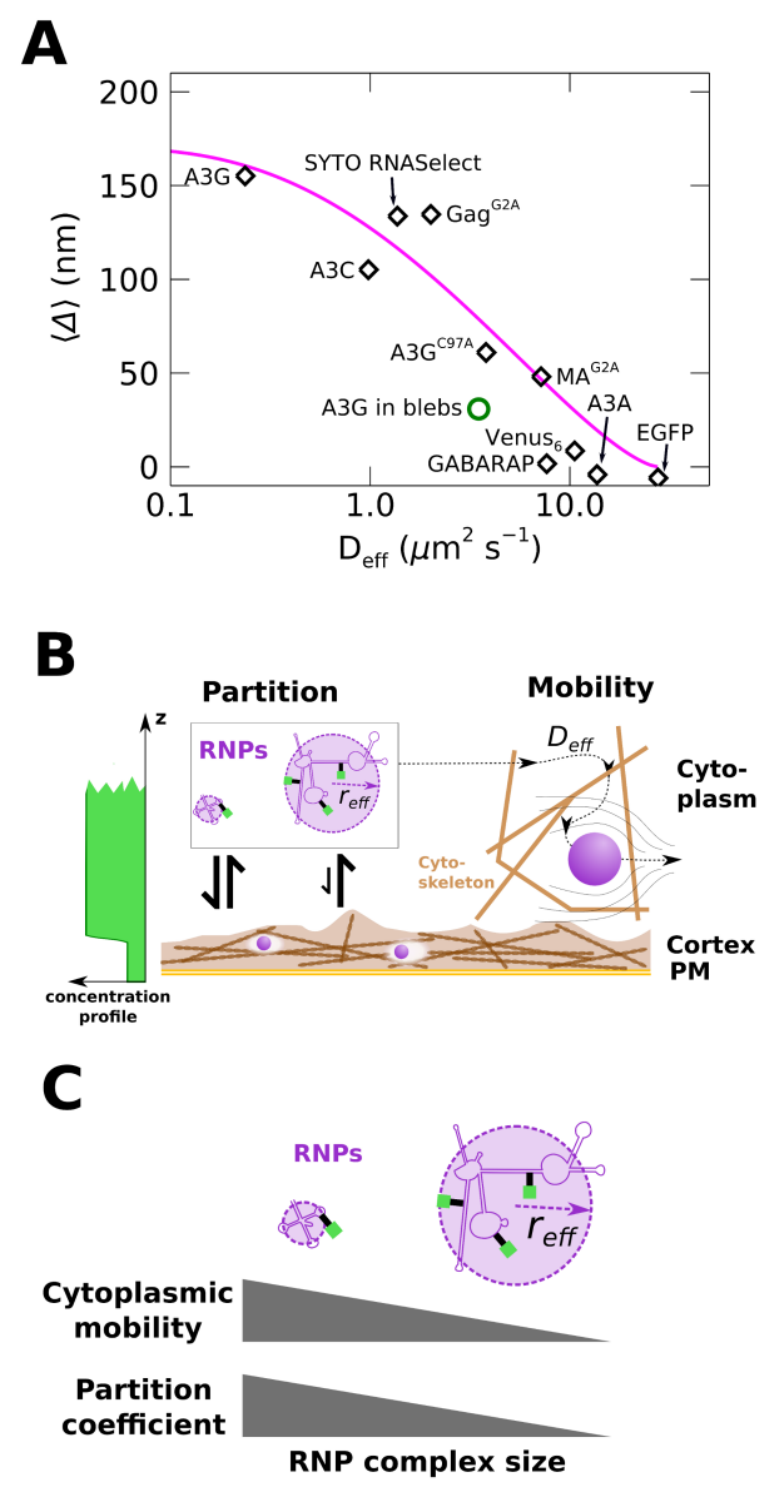

Fig. 6: Protein mobility and effective depletion length. A) Depletion length $<\Delta>$ versus effective diffusion

coefficient $D_{\text {eff }}$ in HeLa cells (diamonds). Eq. 8 (magenta curve) is plotted with parameter values as

911 described in the text. A3G-EGFP measured in HeLa cell blebs (green open circle) displayed increased

912 mobility along with decreased effective depletion length. B) Conceptual model of the effects quantified in

913 panel A. The partitioning effect (center left) arises due to hindered entry of large RNP complexes into the

914 cortical mesh, while hindered mobility of large RNP complexes due to macromolecular crowding occurs in

915 the cytoplasm (right). Binding of labeled proteins to partitioned RNP complexes yields the concentration

916 gradient (far left) observed by DC z-scan measurements. C) The cytoplasmic mobility and partition

917 coefficient are connected by the average RNP complex radius, as indicated in the graphical depiction and

918 suggested by Eqs. 7 and 8. 